\title{
Efecto de la Educación sobre el Delito: Evidencia para Argentina*
}

\author{
Carolina Lopez $z^{\dagger}$ \\ Tesis de Maestría \\ Maestría en Economía \\ Universidad Nacional de La Plata \\ Directora: María Laura Alzúa
}

\begin{abstract}
Resumen
Este trabajo provee evidencia empírica sobre el efecto causal de la educación en los niveles de delitos de Argentina. Para ello se emplea una estrategia de identificación basada en la metodología de diferencias en diferencias. En el análisis se explota la implementación de la Ley Federal de Educación, la cual aumentó los años de educación obligatorios. Es posible utilizar la metodología mencionada ya que la adopción de la reforma no fue simultánea entre las provincias, como así tampoco su intensidad. Los resultados obtenidos indican que el aumento en los años de educación generados por la reforma ocasionó caídas significativas en las tasas del total de delitos y los delitos contra la propiedad en particular. No se encontró evidencia del impacto de la educación sobre los delitos contra las personas y los homicidios dolosos, resultado consistente con el modelo canónico del delito (Becker, 1968). La principal conclusión del trabajo es que la evidencia presentada sugiere considerar a la inversión en la educación como un instrumento de suma relevancia para combatir al delito en el largo plazo.
\end{abstract}

Clasificación JEL: I21, K42.

Palabras Clave: Delitos, Educación, Ley Federal de Educación, Diferencias en diferencias.

\footnotetext{
* Agradezco especialmente a la directora de esta tesis, María Laura Alzúa, por haber sido guía y apoyo constante e incondicional durante todo el desarrollo de este trabajo. Como así también los valiosos comentarios y sugerencias de Guillermo Cruces y Leonardo Gasparini. Los errores y omisiones son de mi exclusiva responsabilidad.

† Centro de Estudios Distributivos, Laborales y Sociales, Facultad de Ciencias Económicas, Universidad Nacional de La Plata. E-mail: clopez@cedlas.org.
} 


\section{Índice}

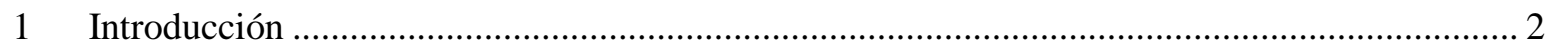

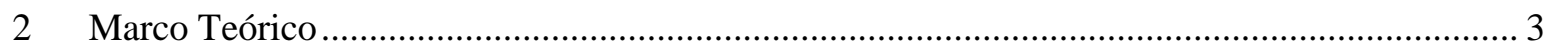

2.1 Canales de transmisión de educación al delito ................................................................ 4

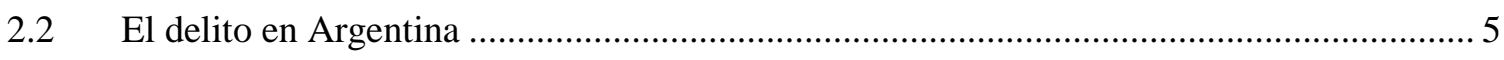

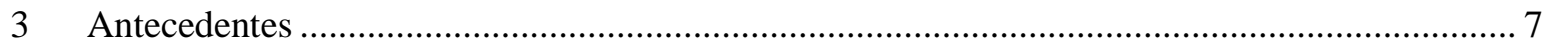

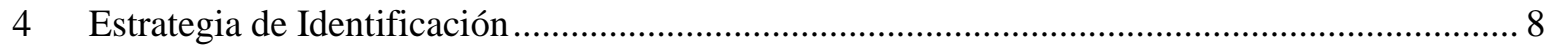

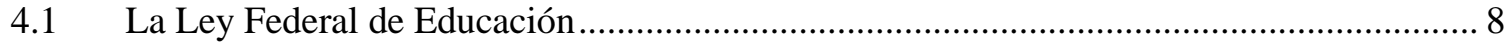

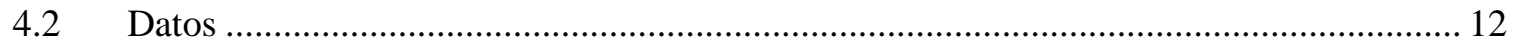

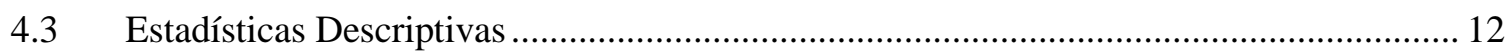

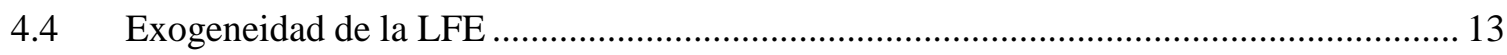

4.5 Estimación del Efecto de la Educación sobre el Delito...................................................... 17

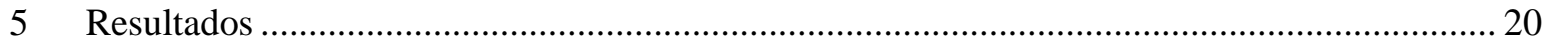

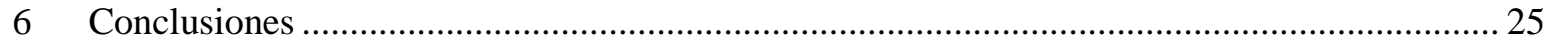

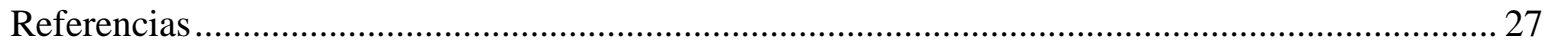

Apéndice A 


\section{Introducción}

El delito es una de las principales preocupaciones de la sociedad (Di Tella, Edwards y Shargrodsky, 2010), por lo que estudiar este fenómeno resulta de gran relevancia al analizar los factores sobre los cuales se puede hacer foco para el diseño de políticas públicas. Numerosos estudios realizados a nivel mundial muestran un hecho en particular: las tasas de delitos registradas en América Latina (tanto robos como homicidios) son las más altas del mundo (por ejemplo Soares y Naritomi, 2010; Bourguignon, 1999; Fajnzylber, Lederman y Loayza, 2002) y dichas tasas regionales presentan gran variabilidad entre países (Di Tella, Edwards y Shargrodsky, 2010).

Entre las causas que pueden explicar tales niveles de delitos pueden mencionarse: la alta desigualdad en la distribución del ingreso, las bajas tasas de arresto y las fuerzas policiales reducidas (Soares y Naritomi, 2010). A pesar de ello, los estudios realizados sobre el delito para la región son escasos. Por este motivo, este trabajo intenta proveer evidencia empírica para Argentina del efecto causal de la educación sobre el delito, factor poco explorado en la literatura de América Latina. Tanto en el corto, mediano o largo plazo, el hecho de extender el acceso a la educación a la mayor población posible, proveerá a los individuos mejores oportunidades en el mercado laboral, y por ello se espera una reducción en la incidencia de los delitos (al aumentar el costo de oportunidad del mismo). No obstante, la literatura sobre esta temática no descarta el hecho de que un mayor nivel educativo puede aumentar el retorno de cometer ciertos delitos más sofisticados, por ejemplo fraudes económicos (Lochner, 2004).

En general la literatura económica analiza el impacto de la educación sobre los retornos privados de los individuos, pero no considerar los efectos de la misma para el resto de la sociedad implica omitir parte de las externalidades positivas que la misma genera. Para analizar el posible impacto de la educación sobre los niveles de delitos en Argentina se emplea una estrategia de identificación que permita obtener el efecto causal buscado, utilizando la introducción de la Ley Federal de Educación, sancionada en 1993, la cual elevó los años de educación obligatorios de 7 a 10 años. Esta ley tuvo cobertura a nivel nacional, sin embargo existieron diferencias en el tiempo de la implementación entre las provincias, de modo que provee variación en la intensidad de su aplicación, por lo que se aplica una estrategia de identificación basada en un experimento natural, generado por la reforma educativa.

Se utilizó una metodología de diferencias en diferencias y los resultados obtenidos indican que la introducción de esta reforma ocasionó caídas significativas en el total de delitos denunciados y los delitos contra la propiedad en particular. No se encontró evidencia del impacto de la reforma sobre los delitos contra las personas y los homicidios dolosos.

El resto de este trabajo se organiza de la siguiente manera. En la Sección 2 se detalla el vínculo del delito con la educación, se presenta un análisis del delito en el país y se explican los canales a través de los cuales la educación puede impactar sobre el delito. En la Sección 3 se realiza una revisión de la literatura relacionada con esta temática. En la Sección 4 se explica la estrategia de identificación empleada, justificando el empleo de la Ley Federal de Educación, se detallan los datos empleados y se plantean los posibles problemas asociados a la metodología utilizada. Posteriormente en la Sección 5 se muestran los resultados obtenidos y finalmente en la Sección 6 se presentan las principales conclusiones de esta investigación. 


\section{Marco Teórico}

De acuerdo a un modelo tradicional del delito (Teoría Económica del Delito, propuesta inicialmente por Becker, 1968), los individuos racionales analizan los costos y beneficios relacionados con cometer o no un delito. La decisión que adopten estos individuos depende principalmente de las posibilidades de generación de ingresos en el sector formal, del castigo por una eventual pena condicionado a la probabilidad de arresto, como así también del poder de disuasión ejercido por la policía (por ejemplo, la cantidad de policías por área geográfica).

Los niveles de pobreza presentes en una sociedad y la desigualdad en la distribución del ingreso, constituyen factores clave en el estudio de los delitos, ya que ante un empeoramiento en las condiciones económicas de un país se generan mayores tensiones sociales, aumenta la desocupación y como así también la cantidad de potenciales criminales. Por estos motivos, es un aspecto fundamental analizar como determinantes de los niveles de delitos otras variables socioeconómicas. Hasta el momento, el análisis económico se centró principalmente en el estudio de las variables relacionadas con el efecto disuasivo del sistema judicial y de la policía (arresto y severidad de la pena) haciendo foco también en los niveles de desigualdad (Kelly, 2000), tratando de resolver de distinta manera los problemas de endogeneidad que surgen al tratar con estas variables ${ }^{1}$.

A pesar de que en América Latina se registraron mejoras en los indicadores de la distribución del ingreso en los años 2000 con respecto a los observados en las décadas pasadas, los niveles de preocupación por la seguridad de la población han ido en aumento (Alzúa, 2011), sobre todo por los altos costos que el delito genera. Estos pueden ser enfocados de distinta manera. En primer lugar, desde la perspectiva de las víctimas, pueden estar asociados a la pérdida material de algún objeto o de daño físico durante el episodio, el cual conlleva gastos de salud, o incluso la muerte. Además, existen costos intangibles que son los que derivan del sufrimiento y del dolor de las víctimas de delitos o de las potenciales víctimas, ya que para evitar los delitos las personas cambian comportamientos que limitan su desarrollo como miembros de una comunidad, dado el entorno en el que viven (Acevedo, 2008). Por otro lado, para evitar ser víctimas de delitos se pueden adoptar medidas de seguridad tales como evitar circular en zonas consideradas peligrosas, no adquirir bienes que los puedan exponer a robos, contratar seguridad privada, etc.. En segundo lugar, para los delincuentes existen costos asociados a la eventual pérdida de la libertad, estar fuera del mercado laboral durante el arresto, deterioro del capital humano adquirido, el estigma asociado a ser un convicto, estar alejado de sus familiares, etc. (crime career costs). Finalmente, desde la perspectiva de los gobiernos, los costos son aquellos asociados a los gastos en los que se debe incurrir en policías, en el sostenimiento del sistema judicial y las unidades carcelarias, como así también en otros tipos de erogaciones que se deben realizar para la contención de las víctimas (gastos del sistema de salud pública, por ejemplo). Estos últimos, de no ser por el nivel de delincuencia podrían ser liberados y dedicados a otros fines más útiles para el desarrollo de un país, como la educación.

\footnotetext{
${ }^{1}$ En el caso de Argentina algunos trabajos incorporan las variables mencionadas, pero no todos logran obtener el efecto causal sobre el crimen por problemas de variables omitidas, causalidad inversa, etc.. Además de las variables disuasivas determinantes del delito (arresto, condena, gasto en seguridad, etc.), estos trabajos consideran otras de carácter social y económico (Kessler y Molinari, 1997; Chambouleyron y Willington 1998; Cerro y Meloni, 1999; Cerro y Rodríguez Andrés, 2011).
} 
Las consecuencias relacionadas a los altos niveles de delitos se traducen en la pérdida de inversiones, la no acumulación de capital humano, pérdida de productividad, etc. con el consecuente impacto negativo futuro en el crecimiento económico de un país (Soares, 2010). Esto se ve agravado por el hecho que los más jóvenes (hombres entre 15 y 24 años) no encuentran oportunidades en el sector formal, y los altos niveles de desempleo para este grupo etario generan una mayor cantidad de potenciales delincuentes (Acevedo, 2008).

Es importante destacar que en un modelo económico del delito, los delitos contra la propiedad son los que más se ajustan al mismo, dado que se cometen por motivaciones económicas. El resto de los delitos contra las personas o los homicidios en particular, pueden estar estrechamente vinculados con los primeros, por lo cual si bien la relación no es tan directa como en el primer caso, se espera que se determinen por las mismas variables que generan los delitos contra la propiedad (Bourguignon, 1999).

Como se ha mencionado, este trabajo analiza el impacto de la educación de los individuos sobre los delitos en Argentina y la importancia del análisis está directamente vinculada a la edad en la que se cometen la mayoría de delitos. En la literatura se evidencia que la mayor proporción de delitos son cometidos por jóvenes (hasta finales de la adolescencia), ya que tienen una menor vinculación con el mercado laboral debido a las pocas posibilidades que el mismo les ofrece. De acuerdo a Acevedo (2008) universalmente, la mayoría de los delitos en la vía pública son cometidos por hombres entre los 15 y 24 años, además se debe tener en cuenta que el perfil edad-salarios es creciente con la edad de los individuos (Grogger, 1998). No obstante, es importante considerar como sostienen diversos criminólogos, que este tipo de patrón en la edad de mayor actividad delictiva no varía a través de razas o etnias, ni siquiera en el tiempo y en las diversas sociedades (Hirschi y Gottfredson, 1984).

En Argentina, de acuerdo a datos del Ministerio de Justicia y Derechos Humanos de la Nación (Dirección Nacional de Política Criminal) en el año 2008, casi el 55 por ciento de los inculpados conocidos por delitos contra la propiedad (hurtos, hurtos de automotores, robos, robos de autos y robos de bancos) son menores de 21 años.

\subsection{Canales de transmisión de educación al delito}

Este trabajo parte de un modelo de capital humano, que predice que mayores niveles educativos conducirán a aumentos en los retornos laborales, lo cual elevará el costo de oportunidad del delito 2 . Este resultado es el esperado en el campo de la Economía del Delito (Becker, 1968), y una posible consecuencia del mismo es que mayor nivel educativo puede conducir a una caída en los niveles de delitos. No obstante, el efecto causal de la educación sobre el mismo fue explorada recientemente (Lochner y Moretti, 2004; Machin, Marie y Vujic, 2010; Meguir, Palme y Schnabel, 2012). El mayor impacto de la educación que se ha analizado en los estudios empíricos se refiere a los retornos privados, tales como el aumento en los salarios o en las mejores oportunidades laborales. Sin embargo, la educación también puede tener retornos sociales positivos, ya que existen canales a través de los cuales los logros educativos individuales pueden afectar la tasa de delitos en una determinada sociedad (Lochner y Moretti, 2004, y Lochner, 2010):

\footnotetext{
${ }^{2}$ El análisis de la relación entre retornos laborales, oportunidades en el mercado laboral y delitos ha sido analizado, por ejemplo, en Grogger (1998) y Machin y Meghir (2004).
} 
- Un mayor nivel educativo produce mayores posibilidades de mejores salarios para los individuos "efecto capital humano", por lo que estar vinculado a actividades delictivas genera un costo de oportunidad alto a mayores años de educación alcanzados.

- La educación puede aumentar la paciencia de los individuos haciendo que valoren más los retornos futuros del trabajo, generando de esta manera que también tengan una mayor aversión con respecto a las posibles condenas que se deben cumplir al cometer un delito.

- Mayores años de educación pueden ocasionar que las preferencias individuales se vean alteradas al estar rodeados de personas más educadas, "efecto pares" (peers). Consecuentemente es menos probable que se cometan delitos. También podría ocurrir que los niños asistan a escuelas en donde se relacionen con otros ya vinculados en actividades delictivas o en situación de riesgo, de modo que el signo de este efecto es ambiguo.

- Permanecer más tiempo en la escuela produce un "efecto incapacitación", ya que estar en las aulas limita la cantidad disponible de horas para vincularse en actividades riesgosas por parte de los jóvenes (Black, Devereux y Salvanes, 2008). Al mismo tiempo aumentan las interacciones entre los adolescentes, que no se ven limitadas solo a los horarios de clase, lo que puede generar que las mismas provoquen comportamientos más violentos entre ellos (Jacob y Lefgren, 2003).

Se espera en general que el efecto capital humano sea el que predomine, siendo la correlación entre el nivel educativo y los delitos negativa. A mayor nivel educativo la probabilidad de los individuos de verse involucrados en actividades delictivas debe ser menor. En este trabajo no se intenta diferenciar cual es el canal de la educación que influye sobre el delito, sino lo que se espera capturar es el efecto neto sobre los mismos. Es importante destacar que no se descarta la posibilidad que mayores años de educación alcanzados aumenten la probabilidad de cometer delitos, ya que pueden incrementar los retornos esperados del mismo al tratarse de crímenes que requieren un alto grado de especialización, además pueden hacer que la detección del delito sea más complicada como así también la probabilidad de arresto (Lochner y Moretti, 2004).

\subsection{El delito en Argentina}

De acuerdo a la encuesta de opinión pública Latinobarómetro (2010) para Argentina, la delincuencia-seguridad pública es el principal problema en el país (37 por ciento de los encuestados). El Laboratorio de Investigaciones sobre Crimen, Instituciones y Políticas (LICIP) de la Universidad Torcuato Di Tella, calcula que en mayo de 2012 la tasa de victimización es del 37.7 por ciento, lo que indica que esta cantidad de hogares de los principales centros urbanos del país han sufrido al menos un delito en los últimos 12 meses (que han sido denunciados o no ante las autoridades competentes), y además que los robos con violencia tienen una participación relativa 56 por ciento del total de delitos (LICIP, Informe Mayo 2012). Estos datos muestran la relevancia del estudio de los delitos en el país.

En el Gráfico $\mathrm{N}^{\mathrm{o}} 1$ se observa la evolución del total de hechos delictuosos, delitos contra la propiedad y las personas en Argentina en el periodo 1992-2008. 


\section{Gráfico 1}

Evolución de Delitos en Argentina 1992-2008: Total de Delitos, Contra las Personas y Contra la Propiedad.

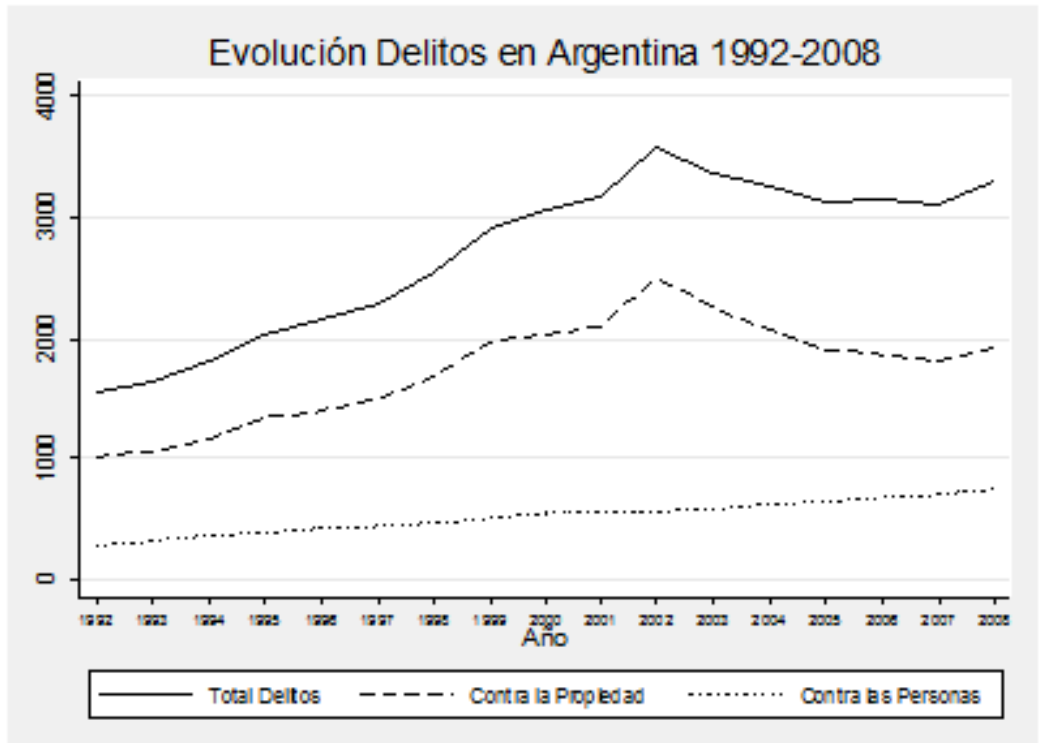

Fuente: Sistema Nacional de Información Criminal (SNIC). Ministerio de Justicia y Derechos Humanos de la Nación. Notas: Delitos denunciados total país. Tasas cada 100 mil habitantes.

Se observa una tendencia creciente del total de delitos desde el año 1992 hasta 2002, en donde se alcanza el máximo nivel de delitos (coincidente con un contexto de crisis económica por la cual atravesaba el país), para luego decrecer hasta 2007 pero manteniéndose en niveles más elevados que en la década de 1990. Dada la importancia relativa de los delitos contra la propiedad, su evolución es la que determina la del total de los delitos en el país.

Es importante destacar que estos datos de delitos provienen de las denuncias realizadas ante las distintas autoridades nacionales y provinciales en cada provincia y la clasificación de los delitos realizada por el SNIC incluye los siguientes tipos:

- Total de hechos delictuosos: delitos contra la propiedad, contra las personas, delitos contra la integridad sexual y el honor (delitos contra el honor, violaciones y otros delitos contra la integridad sexual), delitos contra la libertad (amenazas y otros delitos contra la libertad), delitos contra al Estado y la comunidad (contra la seguridad pública, contra el orden público, contra la seguridad de la Nación, contra los poderes públicos y el orden constitucional, contra la administración pública y contra la fe pública), delitos contra el estado civil, ley 23737 de estupefacientes y otros delitos previstos en leyes especiales.

- Delitos contra la propiedad: robos y tentativas de robo (excluye los agravados por el resultado de lesiones y/o muertes), robos agravados y tentativas de robo agravado por el resultado de lesiones y/o muerte, hurtos y tentativa de hurto, y otros delitos contra la propiedad.

- Delitos contra las personas: homicidios dolosos, homicidios dolosos en grado de tentativa, homicidios culposos en accidentes de tránsito, homicidios culposos por otros hechos, 
lesiones dolosas, lesiones culposas en accidentes de tránsito, lesiones culposas por otros hechos y otros delitos contra las personas.

\section{Antecedentes}

Empíricamente a través de numerosos estudios se ha encontrado que mayores niveles de escolaridad están negativamente correlacionados con el delito, pero los trabajos que captaron el efecto causal son reducidos, aun en países desarrollados. Lochner y Moretti (2004), usando diferentes bases de datos para Estados Unidos emplean en su estrategia de identificación reformas en los sistemas educativos de los estados, que alteran los años de educación obligatorios. Encuentran una significativa disminución en la actividad delictiva a través de la educación, atribuyendo estos cambios a modificaciones en la conducta delictiva. Cuando se emplean datos autoreportados por los jóvenes acerca de su participación delictiva, se encuentra que para los hombres blancos un año adicional de educación reduce su participación en delitos en aproximadamente 1 a 3 puntos porcentuales, mientras que el secundario completo disminuye las tasas de participación en delitos violentos en 9 puntos porcentuales, las ventas de drogas en 5, delitos contra la propiedad en 20 y el total de la participación criminal en 14 puntos porcentuales.

Machin, Marie y Vujic (2010) intentan determinar la relación causal entre la educación y el delito empleando similar estrategia de identificación que Lochner y Moretti (2004), aplicada al caso de Gran Bretaña. Sus resultados muestran que la relación es negativa y significativa, si se disminuye la cantidad de individuos sin educación en un 1 por ciento los crímenes contra la propiedad se reducirían en 1.1 por ciento, indicando que la educación es un canal que puede servir para combatir el delito.

Berthelon y Kruger (2010) analizan para Chile el impacto de una reforma que aumentó la extensión de la jornada escolar sobre la probabilidad de estar vinculados en actividades riesgosas. Las variables de interés son el delito y el embarazo adolescente. Los autores explotan el hecho que la reforma se implementó en forma gradual entre 1997-2006 en las distintas municipalidades, lo cual brindó una estrategia de estimación con diseño no-experimental para determinar la relación entre el efecto incapacitación, generado por un aumento exógeno de las horas de clase, y las conductas riesgosas por parte de los adolescentes. Los resultados obtenidos muestran que la reforma disminuye la probabilidad de embarazo adolescente, como así también el delito juvenil. Con respecto a esta ultima variable se obtiene que ante un aumento en la cobertura de las escuelas con jornada escolar completa en 20 puntos porcentuales, el total de los delitos disminuye en 19 por ciento, los delitos contra la propiedad en 24 y en 11 por ciento los delitos violentos.

Meguir, Palme y Schnabel (2012) analizan los efectos de la introducción de una reforma en el sistema educativo de Suecia, que aumentó los años de educación obligatorios, sobre los delitos para las cohortes afectadas y sus hijos, analizando de esta manera las correlaciones intergeneracionales. Se encuentra un efecto negativo y significativo de la educación sobre los delitos, en el caso de las cohortes directamente afectadas por la reforma, se obtiene que a partir de la misma los delitos se reducen en 1.3 puntos porcentuales, que se corresponde con una disminución del 5 por ciento en la probabilidad de ser condenado. 
Se espera entonces encontrar en este trabajo, y a través de la estrategia de identificación empleada, que la educación tenga un impacto negativo sobre los delitos, como los resultados encontrados por los trabajos previamente mencionados. Sin embargo, la teoría no descarta el hecho que la relación encontrada sea positiva, dado que existen determinados tipos de delitos (como fraudes) que requieren de un nivel educativo alto. En este trabajo se analizan principalmente el total de delitos denunciados, los delitos contra la propiedad, las personas y los homicidios dolosos en particular. Se espera que el impacto sea mayor en los delitos contra la propiedad dado que la motivación subyacente de los mismos es de carácter económico.

\section{Estrategia de Identificación}

En esta investigación se analiza el efecto causal de la educación sobre el delito y las medidas de delitos que se estudian son: el total de hechos delictuosos, los delitos contra la propiedad, contra las personas y los homicidios dolosos, todos ellos medidos en tasas cada 100 mil habitantes.

Una simple regresión de una medida de delito sobre el nivel educativo y otros controles, genera problemas de estimación dado que la educación es endógena, ya que pueden existir factores inobservables que afecten las decisiones de escolaridad que también impacten sobre las decisiones de verse involucrados en actividades criminales. Por ejemplo, una mayor tasa de descuento implica individuos más impacientes en cuanto a los retornos que pueden obtener en el mercado laboral, lo que puede generar que decidan no educarse más (Lochner y Moretti, 2004). Además esta alta tasa de descuento hace que no les preocupe tanto la probabilidad de tener que cumplir una condena, por lo que le parecerá más atractivo el delito. De modo que puede existir una correlación negativa entre las variables de interés, aun cuando la educación no impacte sobre el delito.

Entonces, para obtener el efecto causal de la educación sobre el delito, se debe emplear una estrategia de identificación confiable. Para ello en este caso, se emplea la Ley Federal de Educación ${ }^{3}$ (LFE), como una fuente de variación exógena de los años de educación.

Las leyes que promueven la cantidad de años obligatorios que una persona debe permanecer en la escuela, han tenido un significante impacto en los logros educativos de los individuos (Lochner y Moretti, 2004). En el caso de Argentina Alzúa et al. (2010) concluyen que la Ley Federal de Educación tuvo efectos positivos tanto en el nivel educativo como laboral de los jóvenes que fueron alcanzados por la reforma, siendo más significativos en el caso de los jóvenes no pobres. Pero más allá de los beneficios privados generados por la mayor educación es importante analizar los retornos sociales, entre ellos la posible reducción en los niveles de delitos (Lochner y Moretti, 2004), dado que el delito es una externalidad negativa reducir sus niveles conlleva a una mejora para toda la sociedad.

\subsection{La Ley Federal de Educación}

Esta reforma se sancionó en Argentina en el año 1993 en el marco de una serie de medidas tomadas por el gobierno nacional para transferir a las provincias la provisión, administración y financiamiento de las escuelas secundarias, a partir de las cuales cada provincia debía decidir el

${ }^{3}$ Ley N $\mathrm{N}^{\mathrm{o}} 24195$, sancionada el 14 de Abril de 1993 y promulgada el 29 de abril de 1993. 
gasto asignado a la educación, como así también las decisiones salariales del personal docente, no docente y la determinación del año escolar (Crosta, 2007; Galiani y Shargrodsky, 2002) ${ }^{4}$.

El principal objetivo de la LFE fue disminuir los niveles de deserción escolar garantizando el acceso a la educación básica de todos los habitantes (Artículo 3). La misma fue aprobada en un periodo en el que se llevaban grandes cambios en el país, y la educación no fue la excepción (Alzúa et al., 2010).

Uno de los grandes cambios que produjo esta reforma en el sistema educativo fue la obligatoriedad de la educación pre-primaria a los 5 años, el aumento de la educación primaria de 7 a 9 años (Educación General Básica, EGB) y la educación secundaria pasó de 5 a 3 (o 4) años y constaba de un ciclo de especialización (Polimodal). En total los años de educación obligatorios que un niño afectado totalmente por la reforma debía cursar alcanzó los 10 años. En el Cuadro A.1 del Apéndice A se muestran las equivalencias y los años obligatorios entre el antiguo sistema educativo y el que contemplaba esta reforma educativa. Además, esta reforma educativa implicaba cambios en la estructura curricular de las materias a través de todos los ciclos de la primeria y secundaria.

La LFE en Argentina implicaba principalmente que la obligatoriedad de años de educación se extendía a los dos primeros años del nivel secundario, estimándose la edad de los niños afectados entre 13 y 15 años $^{5}$. En el Gráfico 2 se muestra para los principales 16 aglomerados definidos por la Encuesta Permanente de Hogares (EPH) en Argentina, la tasa de enrolamiento entre 1992 y 2002 para niños entre 6 a 12 años y los comprendidos entre 13 y 15 años. Se observa para el primer grupo que la tasa de asistencia es casi total, pero la misma en el caso de los niños entre 13 y 15 años no supera el 87 por ciento en los años previos a la implementación de la reforma y que aumenta posteriormente a la adopción de la misma.

\footnotetext{
${ }^{4}$ Ley $\mathrm{N}^{\circ} 24049$, Descentralización educativa.

${ }^{5}$ En Argentina se estima que un niño entre 6 y 12 años esté cursando el nivel primario de educación (Cuadro A.1 del Apéndice A). Pero no se descarta la posibilidad que mayores permanezcan en la escuela debido a la repitencia de grados, lo cual hace que el umbral de 12 no sea fijo.
} 


\section{Gráfico 2}

Tasas de Enrolamiento 1992-2002 por Grupo Etario. Principales Aglomerados de Argentina.

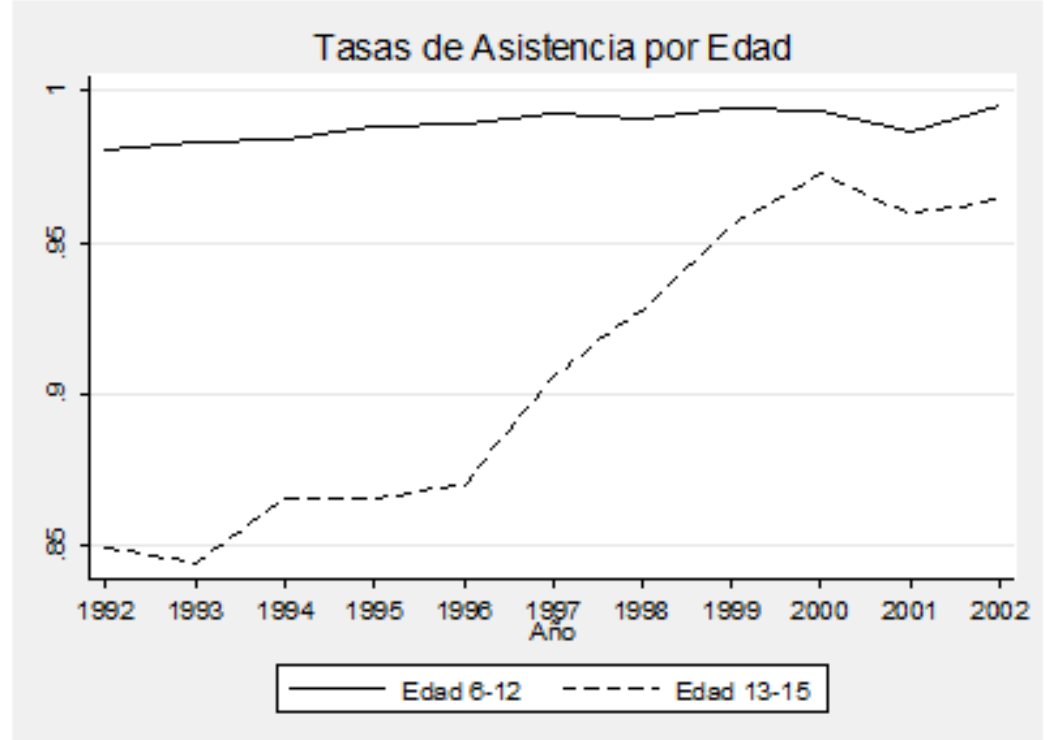

Fuente: Elaboración propia en base a Alzúa et al. (2010), sobre la Base de Datos Socioeconómicos para América Latina y el Caribe-SEDLAC, CEDLAS y Banco Mundial, (2012). Nota: Los aglomerados considerados son aquellos para los cuales hay información en todo el periodo considerado.

Para respaldar la estrategia de identificación que se emplea en este trabajo, en el Gráfico 3 se muestra la evolución de las tasas de enrolamiento de los jóvenes entre 13 a 15 años entre los años 1992 y 2002, para el grupo de provincias que implementaron la reforma de manera temprana (definido como los que adoptaron la reforma entre 1996 y 1997) y para aquellas que lo hicieron de manera tardía o nunca la implementaron (definido como los que implementaron luego de 1997).

Puede observarse que las tasas de enrolamiento, tuvieron una tendencia creciente hasta 1996, año en que las provincias comenzaron a implementar la LFE. A partir de ese año se evidencia que las tasas de asistencia en las provincias que adoptaron la reforma rápidamente aumentaron de modo tal que alcanzaron los niveles de las que no lo hicieron o lo realizaron de manera tardía. Por lo que este gráfico muestra evidencia de carácter visual de que las provincias en ambos grupos (entendiéndose en este caso a las que aplicaron la ley con más antelación que el resto) compartían una tendencia subyacente común, pero como consecuencia de la implementación de la reforma aquellas que lo hicieron de manera temprana tienen un desvío de la tendencia previa, originada en principio por el efecto de la reforma. 


\section{Gráfico 3}

Tipo de Implementación de la LFE. Niños entre 13 y 15 años. Principales Aglomerados de Argentina.

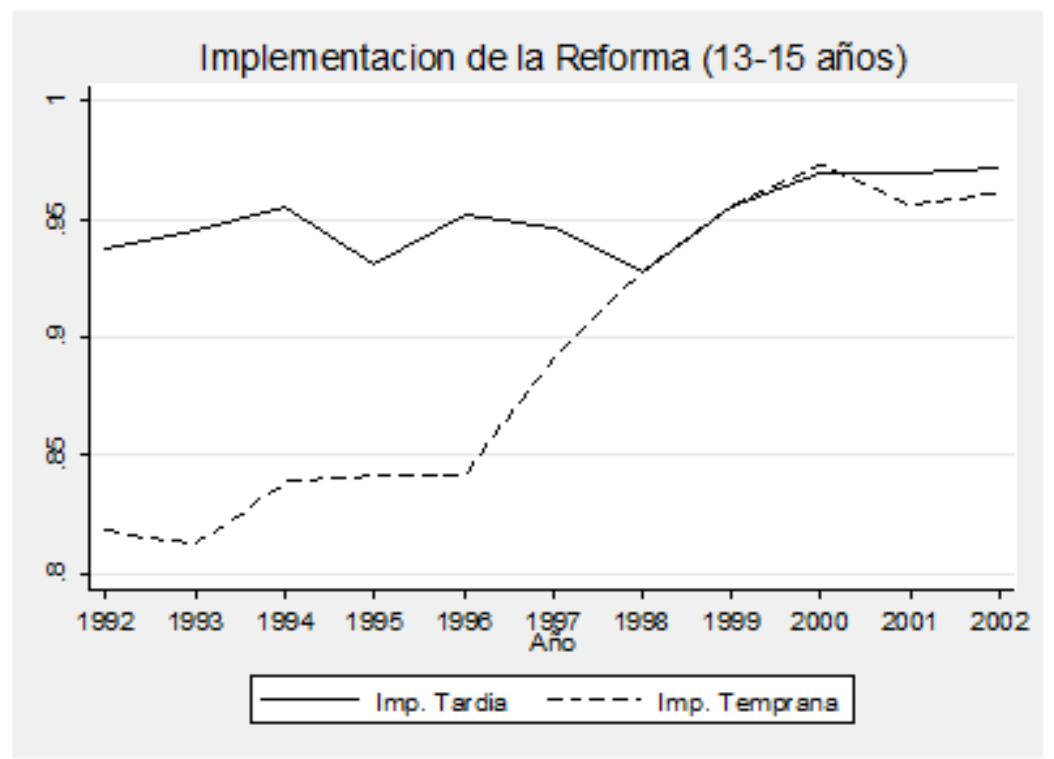

Fuente: Elaboración propia en base a Alzúa et al. (2010), sobre la Base de Datos Socioeconómicos para América Latina y el Caribe-SEDLAC, CEDLAS y Banco Mundial, (2012). Nota: Los aglomerados considerados son aquellos para los cuales hay información en todo el periodo considerado.

Como ya se señaló, la LFE se aplicó en distintos momentos de tiempo a fines de la década de 1990 y a principios de 2000 en casi todas las provincias de Argentina, pero quienes nunca la implementaron en sus aspectos esenciales fueron la provincia de Río Negro y la Ciudad Autónoma de Buenos Aires (Crosta, 2009). Además, es importante considerar que el grado de exposición aumenta cuando más jóvenes son los niños afectados por la reforma.

Pero en este punto es importante destacar que, como se señaló previamente, la intensidad con la que se aplicó la ley fue diferente entre las provincias que la adoptaron, por ejemplo Neuquén la implementó en 1998 pero en el año 2002 solo 6 de un total de 101 establecimientos de educación media dictaban el Polimodal. De este hecho surge la necesidad de contar con distintas medidas de la intensidad de la aplicación de la LFE. En este trabajo se emplea la estrategia seguida por Crosta (2009) que usa la cantidad de años hasta 2000 desde la implementación de la reforma en cada provincia, la cantidad de establecimientos en donde se imparten los ciclos establecidos por la LFE (en este trabajo se analiza en particular el Polimodal) y una medida de adopción temprana o tardía, como opciones al grado de exposición de la ley (intensidad).

Dado que en esta investigación se intenta determinar el impacto de la educación sobre los niveles de delitos, empleando en la estrategia de identificación principalmente a la LFE, es importante destacar el efecto que tuvo esta ley sobre el nivel educativo. A modo descriptivo se observa que para las 15 principales ciudades de Argentina en el año de la aprobación de la ley (1993) el porcentaje de jóvenes en edad de asistir al nivel secundario y que efectivamente asisten es del 64.48 por ciento, mientras que dos años después del comienzo de la implementación de la misma (1998) este porcentaje llega al 80.34 por ciento. Al emplear una estrategia de identificación que explota la variación en la intensidad del tratamiento, Alzúa et al. (2010) obtienen que aquellos que fueron 
expuestos a la reforma tienen más años de educación y mayor probabilidad de terminar con la educación media, aunque el efecto encontrado es mayor para aquellos jóvenes no pobres. Con respecto al desempeño educativo, utilizando datos de los Operativos Nacionales de Evaluación (ONE) se encuentra un efecto positivo de la reforma en los scores alcanzados en Lengua, pero no hay efectos significativos en los de Matemáticas. Berlinsky, Galiani y Gertler (2006) analizan el impacto de la obligatoriedad del último año del nivel inicial, propuesto por la LFE, sobre logros académicos en la educación primaria. Se estima que existe un efecto positivo de la expansión de las facilidades en el acceso a este nivel inicial sobre las evaluaciones estandarizadas de Lengua y Matemáticas en tercer grado de la escuela primaria.

\subsection{Datos}

Para estudiar el impacto de los años de educación sobre las tasas de delitos se emplearán datos a nivel provincial del total de hechos delictuosos, delitos contra la propiedad y contra las personas, y en particular los homicidios dolosos. Se cuenta con información para los años 1992 a 2008 de estos hechos delictivos, y los datos provienen de los informes anuales del Sistema Nacional de Información Criminal (SNIC) de la Dirección Nacional de Política Criminal del Ministerio de Justicia y Derechos Humanos de la Nación. Es importante destacar que estos datos constan de las denuncias realizadas por las víctimas, si bien se sabe que existe un subreporte de las mismas y que varía de acuerdo al tipo de delito, es usual en esta literatura trabajar con estos datos, ya que estas tasas se mantienen relativamente estables con los ciclos económicos.

Para obtener información de características demográficas por provincias, se emplearán las bases elaboradas por el SEDLAC Base de Datos Socioeconómicos para América Latina y el Caribe, un proyecto de CEDLAS - Universidad Nacional de La Plata y el Grupo de Pobreza y Género del Banco Mundial (LCSPP). La base SEDLAC contiene información de más de 300 encuestas de hogares nacionales en 25 países de América Latina y el Caribe. Todas las variables en SEDLAC están construidas usando criterios consistentes entre países y años, aplicando las mismas rutinas de procesamiento (ver sedlac.econo.unlp.edu.ar).

Los datos de la implementación de la reforma por provincias provienen de la Dirección Nacional de Información y Evaluación de la Calidad Educativa (DINIECE) del Ministerio de Educación de la Nación. La información con respecto a la evolución de los gastos provinciales proviene de la Dirección Nacional de Política Macroeconómica del Ministerio de Economía y Finanzas Públicas de la Nación (MECON).

\subsection{Estadísticas Descriptivas}

A fin de analizar las variables de interés en esta investigación, como así también las que se consideran a través de todo el análisis, en el Cuadro 1 se realiza un análisis descriptivo de las mismas pero considerando dos agrupamientos de años: uno de ellos se considera que incluye tasas de delitos no afectadas por la reforma y comprende los años 1992 a 2000, mientras que el segundo incluye aquellos años con tasas de delitos consideradas como alcanzadas por la misma (un análisis detallado de porque se consideran tales grupos se realiza en la Sección 4.5). 
Cuadro 1

Estadísticas Descriptivas

\begin{tabular}{|c|c|c|c|c|c|c|c|c|c|c|}
\hline \multirow{2}{*}{ Variables } & \multicolumn{5}{|c|}{ Años no afectados por la Reforma: $1992-2000$} & \multicolumn{5}{|c|}{ Años afectados por la Reforma: 2003-2008 } \\
\hline & Media & SD & $\mathrm{N}$ & Mínimo & Máximo & Media & SD & $\mathrm{N}$ & Mínimo & Máximo \\
\hline Tasa hechos delictuosos & 2440.45 & 1096.11 & 213 & 923.00 & 7439.00 & 3645.62 & 1347.13 & 144 & 1612.41 & 7282.67 \\
\hline Tasa contra las personas & 470.07 & 206.68 & 213 & 126.00 & 1337.00 & 702.43 & 273.63 & 144 & 246.36 & 1449.59 \\
\hline Tasa contra la propiedad & 1637.44 & 824.17 & 213 & 508.00 & 4860.00 & 2304.80 & 1010.07 & 144 & 748.58 & 5158.65 \\
\hline Tasa homicidios dolosos & 9.02 & 8.14 & 212 & 0.50 & 44.94 & 5.01 & 2.32 & 144 & 0.77 & 13.60 \\
\hline Años promedio de educación & 7.27 & 0.72 & 159 & 5.80 & 9.91 & 8.11 & 0.61 & 141 & 7.00 & 10.62 \\
\hline Años promedio de educación (15-24 años) & 9.96 & 0.56 & 159 & 8.18 & 11.88 & 10.45 & 0.42 & 141 & 9.64 & 12.07 \\
\hline Tasa de asistencia escolar (6-12 años) & 0.99 & 0.01 & 159 & 0.96 & 1.00 & 0.99 & 0.01 & 141 & 0.93 & 1.00 \\
\hline Tasa de asistencia escolar (13-15 años) & 0.92 & 0.04 & 159 & 0.79 & 0.99 & 0.95 & 0.03 & 141 & 0.83 & 1.00 \\
\hline Gasto en educación básica & 307.07 & 445.69 & 192 & 56.09 & 2930.60 & 845.27 & 1488.21 & 144 & 96.22 & 12536.14 \\
\hline Tasa de desocupación & 0.11 & 0.04 & 159 & 0.02 & 0.21 & 0.08 & 0.04 & 141 & 0.01 & 0.18 \\
\hline Tasa de desocupación (15-24 años) & 0.23 & 0.08 & 159 & 0.06 & 0.43 & 0.19 & 0.08 & 141 & 0.01 & 0.42 \\
\hline Tasa de pobreza a 2.5 US\$ & 0.13 & 0.08 & 159 & 0.01 & 0.36 & 0.16 & 0.10 & 141 & 0.01 & 0.45 \\
\hline Desigualdad Gini & 0.45 & 0.03 & 159 & 0.38 & 0.55 & 0.46 & 0.03 & 141 & 0.38 & 0.56 \\
\hline Índice de masculinidad (15-24 años) & 98.25 & 8.27 & 159 & 71.04 & 119.49 & 101.35 & 9.25 & 141 & 81.40 & 131.22 \\
\hline
\end{tabular}

Fuente: Elaboración propia sobre la Base de Datos Socioeconómicos para América Latina y el CaribeSEDLAC, CEDLAS y Banco Mundial (2012), MECON y SNIC. Notas: las tasas de delitos se reportan cada 100 mil habitantes. Para el año 1998 el SNIC no reporta datos sobre delitos para las provincias de Salta y Jujuy, por lo que se les imputó los del año 1999. Gasto en educación básica en pesos millones de pesos de 2001.

Puede observarse que para los años con tasas de delitos afectadas por la reforma las tasas de total de hechos delictuosos, delitos contra las personas y contra la propiedad, eran en promedio menores con respecto a los años que si fueron afectados por la LFE (este patrón puede observarse en el Gráfico 1), mientras que en el caso particular de los homicidios dolosos el promedio a través de las provincias consideradas es mayor en los años considerados no afectados por la reforma. Con respecto a las variables educativas se observa que los años de educación promedio para el total de la población crecieron en promedio 0.84 años en los años posteriores a las implementación de la ley. Restringiendo este cálculo al grupo etario entre 15 y 24 años se observa que, en promedio, los años de educación aumentaron en 0.49 años. Las tasas de asistencia para los niños en edad de asistir a la escuela primaria permaneció casi universal entre estos años (como se observa en el Gráfico 2), mientras que para aquellos jóvenes entre 13 y 15 años se registró en promedio un leve aumento de 0.03 años. Con respecto al gasto total en educación básica por parte de los gobiernos provinciales aumentó en promedio. Los niveles de desocupación en promedio cayeron levemente (considerando el total de la población y a los jóvenes entre 15 y 24 años). Los niveles de pobreza aumentaron en los años afectados por la reforma, mientras que la desigualdad lo hizo levemente. La inclusión del índice de masculinidad, que indica la cantidad de hombres cada 100 mujeres comprendidos entre 15 y 24 años, se justifica en el hecho que cuanto mayor es la población con estas características se eleva el número de potenciales criminales en la sociedad (Acevedo, 2008). Esta variable aumentó en promedio con respecto a los años previos a la reforma.

\subsection{Exogeneidad de la LFE}

En este trabajo se explota el hecho que si bien la LFE tuvo cobertura nacional, su implementación fue gradual y progresiva entre las provincias, de modo que esto provee variación temporal a nivel provincial, en la intensidad de la aplicación de la ley. De acuerdo al texto de la misma y al contexto 
en el cual se sancionó, no existen indicios que indiquen que la LFE se haya adoptado para reducir o alterar los niveles de delitos en el país. Pero no se puede excluir la posibilidad que los niveles de delitos no estén correlacionados con la implementación de la LFE a través de las provincias.

Es importante destacar que para poder adoptar la reforma, cada provincia requería mayores partidas presupuestarias por parte de la Nación, ya que se debía hacer frente a una mayor cantidad de erogaciones para la construcción escuelas o la ampliación de las existentes y una mayor dotación de docentes, dado que la cantidad de niños en las escuelas aumentaría como consecuencia del incremento de los años obligatorios. Con respecto a este punto, si la decisión de la implementación de la ley por parte de los gobiernos provinciales se relaciona con factores que a su vez afecten las variables de interés, en este caso las tasas de delitos, puede generar que la estrategia de identificación resulte no válida al ser endógena la LFE (Alzúa et al. 2010). Si las provincias que decidieron adoptar la reforma por una mayor necesidad de fondos nacionales, al tener escasos recursos, y si a su vez las mismas registran elevadas tasas de delitos, las estimaciones obtenidas pueden sufrir de sesgos.

Para testear esta fuente de posible sesgo, se sigue la estrategia planteada por Berthelon y Kruger (2010), que estiman una regresión del porcentaje de implementación de una reforma que aumentó la jornada escolar en Chile, sobre variables que influyeron en la decisión por parte de los municipios. En este caso se estima una regresión como la siguiente:

$$
\text { ImplPolimodal }_{i t}=\gamma+\delta X_{i t}+\rho_{t}+\mu_{i}+v_{i t}
$$

En donde ImplPolimodal $_{i t}$ es el porcentaje de implementación del nivel polimodal en la provincia $i$ en el año $t$. Se incluye esta medida como una proxy de implementación de la LFE, ya que la adopción fue progresiva entre las provincias y la implementación de este nivel indicaría un alto grado de adopción de la misma. $X_{i t}$ incluye una medida de pobreza, de desigualdad, de desocupación, gasto en educación básica por parte de los gobiernos provinciales, tasa de asistencia a la escuela de los niños que deberían cursar el tercer ciclo de EGB y un índice de masculinidad para los jóvenes entre 15 y 24 años. Pero además este vector de variables incluye las tasas de delitos que se desean estudiar, pero se las incorpora de manera separada. Se consideran también efectos fijos por provincia y por año, y $v_{i t}$ es el término del error.

Los resultados se muestran en el Cuadro 2, en donde se observa una estimación por cada variable de interés. Se evidencia que en todos los casos analizados, las provincias con mayores tasas de enrolamiento en EGB 3 tienen una mayor proporción de implementación del nivel Polimodal en establecimientos de educación media. La variable que genera una mayor preocupación en cuanto a la introducción de sesgos, el nivel de pobreza en cada provincia, no resulta estadísticamente significativa en ninguna de las estimaciones realizadas. En el caso de las tasas del total de hechos delictuosos, contra las personas y la propiedad, no se encuentra evidencia estadísticamente significativa de que tales tasas hayan influido en la implementación del Polimodal en cada provincia. Solo para la tasa de homicidios dolosos se encuentra significatividad estadística al 10 por ciento, dadas el resto de las variables incluidas en el modelo. 
Cuadro 2

Determinantes de la Implementación del Nivel Polimodal

\begin{tabular}{|c|c|c|c|c|}
\hline & \multicolumn{4}{|c|}{ Porcentaje de Implementación de Polimodal } \\
\hline & (1) & $(2)$ & (3) & (4) \\
\hline Tasa hechos delictuosos & $\begin{array}{c}0.0001 \\
{[0.0001]}\end{array}$ & & & \\
\hline Tasa contra las personas & & $\begin{array}{c}0.0001 \\
{[0.0006]}\end{array}$ & & \\
\hline Tasa contra la propiedad & & & $\begin{array}{c}0.0001 \\
{[0.0001]}\end{array}$ & \\
\hline Tasa homicidios dolosos & & & & $\begin{array}{c}0.0443 \\
{[0.0249]^{*}}\end{array}$ \\
\hline Tasa de pobreza 2.5 US\$ & $\begin{array}{c}0.4166 \\
{[0.8100]}\end{array}$ & $\begin{array}{c}0.4030 \\
{[0.8385]}\end{array}$ & $\begin{array}{c}0.4209 \\
{[0.8010]}\end{array}$ & $\begin{array}{c}0.3534 \\
{[0.8443]}\end{array}$ \\
\hline Tasa de desocupación & $\begin{array}{c}-0.6486 \\
{[2.1454]}\end{array}$ & $\begin{array}{c}-0.5491 \\
{[2.0230]}\end{array}$ & $\begin{array}{c}-0.7830 \\
{[2.2113]}\end{array}$ & $\begin{array}{l}-1.0650 \\
{[2.0576]}\end{array}$ \\
\hline Log. gasto educación básica & $\begin{array}{l}-0.3001 \\
{[0.3305]}\end{array}$ & $\begin{array}{l}-0.3346 \\
{[0.3418]}\end{array}$ & $\begin{array}{c}-0.3022 \\
{[0.3298]}\end{array}$ & $\begin{array}{c}-0.3042 \\
{[0.3605]}\end{array}$ \\
\hline Desigualdad Gini & $\begin{array}{c}0.0272 \\
{[1.3915]}\end{array}$ & $\begin{array}{c}-0.1394 \\
{[1.4407]}\end{array}$ & $\begin{array}{c}-0.0031 \\
{[1.3579]}\end{array}$ & $\begin{array}{l}-1.3576 \\
{[1.2242]}\end{array}$ \\
\hline Tasa de asistencia (13-15 años) & $\begin{array}{c}2.2855 \\
{[0.8979]^{* *}}\end{array}$ & $\begin{array}{c}2.5428 \\
{[0.8936]^{\star * *}}\end{array}$ & $\begin{array}{c}2.1806 \\
{[0.9313]^{\star *}}\end{array}$ & $\begin{array}{c}2.0234 \\
{[0.9285]^{\star *}}\end{array}$ \\
\hline Índice de masculinidad (15-24 años) & $\begin{array}{c}0.0033 \\
{[0.0038]}\end{array}$ & $\begin{array}{c}0.0033 \\
{[0.0048]}\end{array}$ & $\begin{array}{c}0.0034 \\
{[0.0039]}\end{array}$ & $\begin{array}{c}0.0038 \\
{[0.0037]}\end{array}$ \\
\hline Constante & $\begin{array}{c}4.0136 \\
{[7.3299]}\end{array}$ & $\begin{array}{c}4.2876 \\
{[7.6449]}\end{array}$ & $\begin{array}{c}4.1802 \\
{[7.2869]}\end{array}$ & $\begin{array}{c}5.0109 \\
{[7.7547]}\end{array}$ \\
\hline Observaciones & 208 & 208 & 208 & 207 \\
\hline $\mathrm{R} 2$ & 0.77 & 0.76 & 0.77 & 0.79 \\
\hline
\end{tabular}

Notas: Tasas de delitos cada 100 mil habitantes. Errores estándar agrupados a nivel provincial entre corchetes. Todas las especificaciones incluyen efectos fijos por provincia y por año. Ponderación por población provincial.

* significativo al 10\%; ** significativo al 5\%; *** significativo al $1 \%$

Dado que no se pueden descartar, a pesar de los resultados previos, problemas en la exogeneidad de la implementación LFE, en este trabajo también se emplea la metodología seguida por Alzúa et al. (2010), en donde se estimaron hazard models para determinar si factores observables afectaron simultáneamente las variables laborales y educativas de interés y la probabilidad de implementar esta reforma educativa en Argentina. Los resultados que obtienen indican que si una provincia es gobernada por el mismo partido que el nacional, es más probable que implemente la ley. Como se mencionó al principio de esta sección, existen otros posibles problemas asociados a la estrategia de identificación empleada, que están relacionados con las características de las provincias de Argentina. Tal como se mencionó previamente, para adoptar la reforma los distritos debían aumentar la infraestructura escolar (dado que debían contener mayor cantidad de alumnos), por lo que mayores partidas presupuestarias eran necesarias. De este modo resulta posible que las provincias más pobres (a priori con mayores niveles de delitos) hayan implementado la LFE para recibir mayor asignación presupuestaria. 
Cuadro 3

Hazard Model Implementación del 90 por ciento de Polimodal

\begin{tabular}{|c|c|c|c|c|c|c|c|c|}
\hline & \multicolumn{8}{|c|}{ Implementación de la Reforma $90 \%$ de Polimodal } \\
\hline & (1) & (2) & (1) & (2) & (1) & (2) & (1) & (2) \\
\hline Tasa hechos delictuosos & $\begin{array}{c}-0.0002 \\
{[0.0003]}\end{array}$ & $\begin{array}{c}-0.0004 \\
{[0.0003]}\end{array}$ & & & & & & \\
\hline Tasa contra las personas & & & $\begin{array}{c}-0.0011 \\
{[0.0026]}\end{array}$ & $\begin{array}{l}-0.0011 \\
{[0.0017]}\end{array}$ & & & & \\
\hline Tasa contra la propiedad & & & & & $\begin{array}{c}-0.0001 \\
{[0.0004]}\end{array}$ & $\begin{array}{c}-0.0004 \\
{[0.0004]}\end{array}$ & & \\
\hline Tasa homicidios dolosos & & & & & & & $\begin{array}{c}0.1874 \\
{[0.1538]}\end{array}$ & $\begin{array}{c}0.1340 \\
{[0.1141]}\end{array}$ \\
\hline Tasa de pobreza 2.5 US\$ & $\begin{array}{c}1.5849 \\
{[4.8534]}\end{array}$ & $\begin{array}{c}1.5957 \\
{[5.1374]}\end{array}$ & $\begin{array}{c}1.2613 \\
{[5.2606]}\end{array}$ & $\begin{array}{c}2.9754 \\
{[5.0554]}\end{array}$ & $\begin{array}{c}2.2158 \\
{[4.8353]}\end{array}$ & $\begin{array}{c}2.5781 \\
{[5.2336]}\end{array}$ & $\begin{array}{c}1.3163 \\
{[5.3415]}\end{array}$ & $\begin{array}{c}3.5706 \\
{[5.0522]}\end{array}$ \\
\hline Partido político & $\begin{array}{c}0.7886 \\
{[0.7791]}\end{array}$ & $\begin{array}{c}0.2745 \\
{[0.7522]}\end{array}$ & $\begin{array}{c}1.0114 \\
{[0.8517]}\end{array}$ & $\begin{array}{c}0.5893 \\
{[0.8445]}\end{array}$ & $\begin{array}{c}0.8388 \\
{[0.7534]}\end{array}$ & $\begin{array}{c}0.2596 \\
{[0.7644]}\end{array}$ & $\begin{array}{c}0.8714 \\
{[0.7951]}\end{array}$ & $\begin{array}{c}0.4672 \\
{[0.8023]}\end{array}$ \\
\hline Tasa de desocupación & $\begin{array}{c}14.9915 \\
{[11.7942]}\end{array}$ & $\begin{array}{c}-6.1109 \\
{[13.5942]}\end{array}$ & $\begin{array}{c}16.2662 \\
{[11.6948]}\end{array}$ & $\begin{array}{c}-2.7969 \\
{[11.8465]}\end{array}$ & $\begin{array}{c}15.1113 \\
{[12.3199]}\end{array}$ & $\begin{array}{c}-5.5480 \\
{[15.5256]}\end{array}$ & $\begin{array}{c}15.9620 \\
{[11.3508]}\end{array}$ & $\begin{array}{c}-0.5455 \\
{[11.8916]}\end{array}$ \\
\hline Log. gasto educación básica & $\begin{array}{c}0.4374 \\
{[0.3171]}\end{array}$ & $\begin{array}{c}1.0505 \\
{[0.6593]}\end{array}$ & $\begin{array}{c}0.3639 \\
{[0.3497]}\end{array}$ & $\begin{array}{c}0.9900 \\
{[0.6678]}\end{array}$ & $\begin{array}{c}0.4843 \\
{[0.3201]}\end{array}$ & $\begin{array}{c}1.0915 \\
{[0.6771]}\end{array}$ & $\begin{array}{c}0.3374 \\
{[0.3010]}\end{array}$ & $\begin{array}{c}0.9146 \\
{[0.5943]}\end{array}$ \\
\hline Desigualdad Gini & $\begin{array}{c}-9.9695 \\
{[22.2868]}\end{array}$ & $\begin{array}{c}-10.0673 \\
{[17.3716]}\end{array}$ & $\begin{array}{l}-12.1018 \\
{[21.7830]}\end{array}$ & $\begin{array}{c}-14.9667 \\
{[18.7190]}\end{array}$ & $\begin{array}{c}-10.8994 \\
\text { [22.9471] }\end{array}$ & $\begin{array}{c}-10.1096 \\
{[18.8571]}\end{array}$ & $\begin{array}{c}-13.4994 \\
{[23.0466]}\end{array}$ & $\begin{array}{l}-14.2900 \\
{[19.4668]}\end{array}$ \\
\hline Años prom. de educación hombres (15-24 años) & $\begin{array}{c}0.2075 \\
{[1.1264]}\end{array}$ & $\begin{array}{c}-0.3281 \\
{[1.5056]}\end{array}$ & $\begin{array}{c}0.2081 \\
{[1.1213]}\end{array}$ & $\begin{array}{c}-0.4642 \\
{[1.7411]}\end{array}$ & $\begin{array}{c}0.0965 \\
{[1.1116]}\end{array}$ & $\begin{array}{c}-0.4633 \\
{[1.4122]}\end{array}$ & $\begin{array}{c}0.2834 \\
{[0.9757]}\end{array}$ & $\begin{array}{c}-0.3500 \\
{[1.7486]}\end{array}$ \\
\hline Tasa de asistencia (13-15 años) & $\begin{array}{c}-0.5492 \\
{[10.7772]}\end{array}$ & $\begin{array}{c}-0.8712 \\
{[15.7755]}\end{array}$ & $\begin{array}{c}-3.3463 \\
{[11.6701]}\end{array}$ & $\begin{array}{c}-5.4516 \\
{[17.1842]}\end{array}$ & $\begin{array}{c}-1.7209 \\
{[10.5454]}\end{array}$ & $\begin{array}{c}-0.7407 \\
{[17.2583]}\end{array}$ & $\begin{array}{l}-5.8270 \\
{[9.7870]}\end{array}$ & $\begin{array}{c}-6.8777 \\
{[17.1591]}\end{array}$ \\
\hline Índice de masculinidad (15-24 años) & $\begin{array}{c}0.0405 \\
{[0.0631]}\end{array}$ & $\begin{array}{c}0.0559 \\
{[0.0437]}\end{array}$ & $\begin{array}{c}0.0396 \\
{[0.0616]}\end{array}$ & $\begin{array}{c}0.0563 \\
{[0.0506]}\end{array}$ & $\begin{array}{c}0.0431 \\
{[0.0643]}\end{array}$ & $\begin{array}{c}0.0546 \\
{[0.0440]}\end{array}$ & $\begin{array}{c}0.0445 \\
{[0.0585]}\end{array}$ & $\begin{array}{c}0.0509 \\
{[0.0513]}\end{array}$ \\
\hline Int & $\begin{array}{c}0.2501 \\
{[0.8584]}\end{array}$ & & $\begin{array}{c}0.3348 \\
{[0.9607]}\end{array}$ & & $\begin{array}{c}0.2128 \\
{[0.8554]}\end{array}$ & & $\begin{array}{c}0.3601 \\
{[0.9868]}\end{array}$ & \\
\hline d2 & & $\begin{array}{c}3.2329 \\
{[2.3582]}\end{array}$ & & $\begin{array}{c}3.0716 \\
{[2.1863]}\end{array}$ & & $\begin{array}{c}3.2015 \\
{[2.4288]}\end{array}$ & & $\begin{array}{c}2.8266 \\
{[2.0689]}\end{array}$ \\
\hline d3 & & $\begin{array}{c}2.7431 \\
{[2.7072]}\end{array}$ & & $\begin{array}{c}2.5361 \\
{[2.3519]}\end{array}$ & & $\begin{array}{c}2.6127 \\
{[2.8057]}\end{array}$ & & $\begin{array}{c}2.0942 \\
{[2.1530]}\end{array}$ \\
\hline d4 4 & & $\begin{array}{c}4.3615 \\
{[2.9169]}\end{array}$ & & $\begin{array}{c}4.0562 \\
{[2.4915]}\end{array}$ & & $\begin{array}{c}4.1418 \\
{[3.0555]}\end{array}$ & & $\begin{array}{c}3.4795 \\
{[2.1905]}\end{array}$ \\
\hline d5 & & $\begin{array}{c}2.8602 \\
{[2.8022]}\end{array}$ & & $\begin{array}{c}2.3236 \\
{[2.2626]}\end{array}$ & & $\begin{array}{c}2.5422 \\
{[3.0816]}\end{array}$ & & $\begin{array}{c}1.6599 \\
{[2.1660]}\end{array}$ \\
\hline d6 & & $\begin{array}{c}4.5096 \\
{[3.7257]}\end{array}$ & & $\begin{array}{c}4.2755 \\
{[3.4011]}\end{array}$ & & $\begin{array}{c}4.3673 \\
{[3.8204]}\end{array}$ & & $\begin{array}{c}3.6484 \\
{[3.4064]}\end{array}$ \\
\hline d7 & & $\begin{array}{c}3.1850 \\
{[2.7653]}\end{array}$ & & $\begin{array}{c}3.0672 \\
{[2.5202]}\end{array}$ & & $\begin{array}{c}3.0403 \\
{[2.7852]}\end{array}$ & & $\begin{array}{c}2.9639 \\
{[2.4564]}\end{array}$ \\
\hline Constante & $\begin{array}{c}-13.8679 \\
{[16.8060]}\end{array}$ & $\begin{array}{c}-20.6448 \\
{[18.8602]}\end{array}$ & $\begin{array}{c}-8.9394 \\
{[16.9646]}\end{array}$ & $\begin{array}{c}-12.7534 \\
{[18.1436]}\end{array}$ & $\begin{array}{c}-12.9388 \\
{[17.4751]}\end{array}$ & $\begin{array}{c}-20.6712 \\
{[21.4854]}\end{array}$ & $\begin{array}{c}-8.4830 \\
{[15.8186]}\end{array}$ & $\begin{array}{c}-12.3993 \\
{[15.9725]}\end{array}$ \\
\hline Observaciones & 145 & 145 & 145 & 145 & 145 & 145 & 144 & 144 \\
\hline
\end{tabular}

Notas: Tasas de delitos cada 100 mil habitantes. Errores estándar agrupados a nivel provincial en corchetes.

(1) Modelo con tendencia temporal; (2) Modelo con dummies de tiempo. Ponderación por población provincial.

* significativo al 10\%; ** significativo al 5\%; *** significativo al $1 \%$

En los hazard models empleados en este caso, se quiere testear si los niveles delictivos influyeron o no en la probabilidad que se adopte la reforma en cada provincia. Para ello se estiman modelos cuyas variables dependientes tienen distintas especificaciones que dependen del porcentaje de la implementación de establecimientos en donde se dicta el nivel Polimodal, sobre el total de establecimientos del antiguo nivel medio. Si una provincia supera ese umbral de implementación entonces se considera que efectivamente aplicó la reforma. Se tomaron tres umbrales del 33 por ciento de implementación, el 66 y el 90 por ciento, y las variables independientes que se consideraron fueron las tasas de delitos analizadas, como así también se incluyeron otras variables a nivel provincial (pobreza, desigualdad, desocupación, índice de masculinidad entre 15 y 24 años, tasa de asistencia entre los 13 y 15 años, años promedio de educación para hombres entre 15 y 24 
años y logaritmo de gastos provinciales en educación primaria $)^{6}$. Dada la significatividad de la variable del partido político gobernante en cada provincia que se obtuvo en Alzúa et al. (2010), se incluye esta variable en los modelos mencionados. Los resultados de emplear un modelo Logit para un umbral de 90 por ciento se muestran en el Cuadro 3. En el caso de los umbrales del 33 y 66 por ciento se muestran en el Apéndice A en el Cuadro A.3 y A.4 respectivamente.

Puede observarse que se plantean dos formas funcionales para modelar la función de riesgo, en la primera de ellas se incluye una tendencia temporal y en la segunda, dummies de años (lo que permite controlar por la implementación de la LFE en el tiempo). Es importante destacar que las dummies de tiempo que se consideran son aquellas en donde se observa que alguna provincia implementa la reforma (de acuerdo al porcentaje considerado), ya que el modelo no puede predecir con respecto a los periodos en los cuales no ocurre el evento. Los resultados indican que cuando se considera un umbral de implementación del 90 por ciento de Polimodal en los establecimientos de educación media, ninguna de las tasas de delitos consideradas resulta estadísticamente significativa, controlando por el resto de las variables incluidas, lo cual es al menos indicativo que las mismas no influyeron en la adopción de LFE por parte de las provincias.

\subsection{Estimación del Efecto de la Educación sobre el Delito}

La estrategia de identificación de esta investigación es similar a la implementada por Duflo (2001). En dicho trabajo se considera el efecto de un aumento en la construcción de escuelas sobre niveles de educación y efectos en el mercado laboral, cuando la asignación de las mismas dependía de las tasas de enrolamiento previas, y en donde la exposición al programa se midió por el lugar y la fecha de nacimiento de las cohortes analizadas. Similar metodología fue empleada por Alzúa et al. (2010), en donde se evaluó el impacto de la Ley Federal de Educación en Argentina sobre resultados laborales y educativos para las cohortes afectadas por la misma. En este trabajo y siguiendo el mismo enfoque se estima una ecuación como la siguiente:

$$
C_{i t}=\alpha+\beta \text { Post }_{t} * \text { Intensidad }_{i}+\theta X_{i t}+\rho_{t}+\mu_{i}+\varepsilon_{i t}
$$

En donde $C_{i t}$ es una medida de delito en la provincia $i$ en el año t (tasas de delitos cada 100 mil habitantes), Post $_{t}$ es una dummy que indica los años en los que se espera que las cohortes afectadas por la reforma tengan participación criminal (los periodos considerados se explican en detalle abajo) y por lo tanto se reflejen en las tasas de delitos consideradas, Intensidad ${ }_{i}$ es una variable que indica el nivel de intensidad de la reforma en la provincia $i, X_{i t}$ en esta ecuación es un vector de regresores que incluye características a nivel provincial, $\rho_{t}$ indica efectos fijos por año, $\mu_{i}$ efectos fijos por provincia y finalmente $\varepsilon_{i t}$ indica el término de error. El supuesto de identificación clave en esta estrategia consiste en suponer que las tendencias en ausencia de la reforma son similares para los grupos de provincias y que no hay variables omitidas que sean provincia-específicas que varíen con el tiempo y que estén correlacionadas con la reforma. Una ventaja de usar la metodología de diferencias en diferencias es la inclusión de un vector de regresores que varíen en el tiempo por

\footnotetext{
${ }^{6}$ Es importante destacar que las provincias de Salta y Jujuy no tienen datos disponibles de delitos en el año 1998, y dado que el modelo comienza a computar la probabilidad de adopción de la reforma en esa fecha (cuando se tiene registro de la proporción de escuelas que cambiaban de sistema) se procedió a imputar la cantidad de delitos del año siguiente para no perder a estas dos provincias en las estimaciones del modelo.
} 
provincias, como una fuente de tendencias provincia-especificas omitidas (Angrist y Pischcke, 2009).

Para respaldar la estrategia de identificación empleada, se plantean dos experimentos: uno de interés y otro de control, siguiendo a trabajos que con anterioridad han utilizado una metodología similar. El primero de ellos consiste básicamente en comparar las medias de las tasas de delitos entre las provincias que implementaron la reforma de acuerdo a la cantidad de años transcurridos desde la implementación hasta el 2000. Esta medida de intensidad de la exposición a la reforma se toma de Crosta (2009). Se considera como altamente expuestas a la reforma, a las provincias que la implementaron de manera generalizada desde el inicio y como baja exposición a las que no la implementaron y a las que lo hicieron pero de manera gradual (ver Cuadro A.2 del Apéndice A).

El período considerado para comparar las tasas de delitos corresponde a uno en que se espera que los jóvenes afectados por la reforma estén en edad activa para comenzar a trabajar (o a delinquir) como se mencionó previamente serían en su mayoría adolescentes. Considerando que un niño de 12 años en 1998, viviendo en una provincia que adoptó tempranamente la ley fue más afectado por la misma que uno que haya nacido en otra provincia que la haya adoptado, por ejemplo, un año después, este niño en el 2006 tendría 20 años (más del 50\% de los delitos contra la propiedad son cometidos por menores de 21 años, Ministerio de Justicia y Derechos Humanos de la Nación, 2008) por lo que aquellos menores de 12 en 1998 fueron aun más expuestos al tratamiento y aun son adolescentes en el 2006. De este modo, se elige analizar las tasas de delitos por provincias en el periodo 2003-2008, en donde estarían en edad de delinquir los que fueron afectados por la reforma ${ }^{7}$. En las estimaciones analizadas se excluye los años 2001 y 2002 por considerarlos como años en los cuales los niveles de delitos fueron extremadamente elevados como consecuencia de la crisis económica-institucional por la cual atravesaba Argentina.

Las tasas de delito que se asumen cometidos por personas no afectadas por la reforma son las previas al año 2000, ya que si en 1998 se implementó la ley en una determinada provincia, un niño de 12 años terminaría de cursar los años obligatorios de educación determinados por la ley en el 2000. Para realizar las diferencias se consideran las tasas de delitos registrados entre el periodo 1992-2000 ${ }^{8}$. Los resultados del experimento de interés se muestran en el Cuadro 4, para cada tipo de delito considerado.

Las tasas de delitos registradas en provincias en donde la reforma se implementó corresponden al periodo 2003-2008 cuando se espera que los jóvenes afectados por la reforma puedan o no delinquir y por lo tanto las tasas registradas estarían reflejando el accionar de los mismos, mientras que los años previos corresponden a los jóvenes mayores que no fueron alcanzados por la ley. Cuando se comparan las medias en provincias donde la exposición a la misma fue alta o baja, la diferencia de las diferencias es -462.38 delitos cada 100 mil habitantes cuando se considera el total de hechos delictuosos y este resultado es estadísticamente significativo al 10\%. En el caso de los delitos contra

\footnotetext{
${ }^{7}$ Es importante destacar que las provincias que implementaron la reforma en 1996, Buenos Aires y Córdoba, lo hicieron de manera gradual, de modo que las cohortes afectadas por la reforma alcanzaron el polimodal en 2001 y 2000 respectivamente (Alzúa et al., 2010).

${ }^{8} \mathrm{Si}$ bien una implementación temprana puede indicar un mayor porcentaje de jóvenes afectados por la reforma, que es lo que se supone en este caso, no siempre puede implicar lo mismo. Además el punto de corte como adoptantes tempranos o tardios puede variar.
} 
la propiedad se encuentra que las diferencias son de -374.18 (estadísticamente significativa al 5\%) y para la tasa de homicidios dolosos y la tasa de delitos contra las personas, las diferencias no resultan estadísticamente significativas. Los resultados obtenidos a través de esta metodología de diferencias en diferencias, pueden ser interpretados como el efecto causal de la LFE sobre los delitos, siempre y cuando se suponga que de no haberse implementado la misma los cambios en las variables de interés analizadas no difieran entre las provincias con alta o baja exposición a la reforma.

Cuadro 4

Diferencias de medias. Experimento de Interés por Tipo de Delito.

\begin{tabular}{|c|c|c|c|c|c|c|}
\hline & \multirow{2}{*}{\multicolumn{3}{|c|}{$\frac{\text { Tasa Total Hechos Delictuosos }}{\text { Exposición a la Reforma }}$}} & \multirow{2}{*}{\multicolumn{3}{|c|}{$\begin{array}{c}\text { Tasa Delitos Contra la Propiedad } \\
\text { Exposición a la Reforma }\end{array}$}} \\
\hline & & & & & & \\
\hline & Alta & Baja & Diferencia & Alta & Baja & Diferencia \\
\hline \multirow[t]{2}{*}{ Años 2003-2008 } & 3074.5042 & 4320.5737 & -1246.0694 & 1869.8682 & 2818.8005 & -948.9323 \\
\hline & [115.1439] & [170.2701] & [491.4977] & [83.5952] & [129.3098] & [366.1999] \\
\hline \multirow[t]{2}{*}{ Años 1992-2000 } & 2097.0598 & 2880.7447 & -783.6849 & 1386.812 & 1961.5638 & -574.7519 \\
\hline & [80.8302] & [123.0965] & [310.6827] & [61.5097] & [92.3897] & [255.8322] \\
\hline \multirow[t]{5}{*}{ Diferencia } & 977.4444 & 1439.829 & -462.3846 & 483.0562 & 857.2366 & -374.1804 \\
\hline & [147.1926] & [201.8628] & [244.2918] & [85.8176] & [140.9163] & [161.2329] \\
\hline & \multicolumn{3}{|c|}{ Tasa Delitos Contra las Personas } & \multicolumn{3}{|c|}{ Tasa Homicidios Dolosos } \\
\hline & \multicolumn{3}{|c|}{ Exposición a la Reforma } & \multicolumn{3}{|c|}{ Exposición a la Reforma } \\
\hline & Alta & Baja & Diferencia & Alta & Baja & Diferencia \\
\hline \multirow[t]{2}{*}{ Años 2003-2008 } & 637.5263 & 779.1377 & -141.6115 & 5.1195 & 4.8788 & 0.2407 \\
\hline & [26.4127] & [36.7691] & [106.1992] & [0.2640] & [0.2861] & [0.8379] \\
\hline \multirow[t]{2}{*}{ Años 1992-2000 } & 427.5812 & 523.9149 & -96.3337 & 9.2891 & 8.6733 & 0.6158 \\
\hline & [16.2773] & [23.6583] & [64.3621] & [0.7825] & [0.8071] & [2.6916] \\
\hline \multirow[t]{2}{*}{ Diferencia } & 209.9451 & 255.2228 & -45.2778 & -4.1697 & -3.7945 & -0.3751 \\
\hline & [35.0005] & [48.0547] & [58.1321] & [1.7159] & [1.7825] & [2.4220] \\
\hline
\end{tabular}

Notas: Errores estándar entre corchetes, para las diferencias errores estándar agrupados a nivel provincial. Tasas de delitos cada 100 mil habitantes. Medida de exposición: Alta si la intensidad de adopción es Full desde el inicio; Baja la si implementación es Gradual o nunca la implementaron (Tabla A.2 del Apéndice A).

Además del experimento de interés se realizó uno de control (Cuadro 5) para verificar que entre aquellos años no expuestos a los efectos de la reforma, no hubieran diferencias significativas entre las provincias que tuvieron alta o baja exposición a la misma. Para ello se consideraron dos periodos no expuestos: el primero es el comprendido entre los años 1996 y 2000 y el segundo entre 1992 y 1995. Los resultados de las dobles diferencias para todas las tasas de delitos considerados no son estadísticamente significativas, lo cual es un indicativo que la estrategia de identificación empleada resulta confiable y se espera que los resultados que se obtengan son debido a la implementación de la LFE (Alzúa et al., 2010). 
Cuadro 5

Diferencias de medias. Experimento de Control por Tipo de Delito.

\begin{tabular}{|c|c|c|c|c|c|c|}
\hline & \multirow{2}{*}{\multicolumn{3}{|c|}{$\begin{array}{c}\text { Tasa Total Hechos Delictuosos } \\
\text { Exposición a la Reforma }\end{array}$}} & \multirow{2}{*}{\multicolumn{3}{|c|}{$\begin{array}{c}\text { Tasa Delitos Contra la Propiedad } \\
\text { Exposición a la Reforma }\end{array}$}} \\
\hline & & & & & & \\
\hline & Alta & Baja & Diferencia & Alta & Baja & Diferencia \\
\hline \multirow[t]{2}{*}{ Años 1996-2000 } & 2354.3077 & 3201.1282 & -846.8205 & 1561.1731 & 2203.8718 & -642.6987 \\
\hline & [115.1439] & [170.2701] & [428.5971] & [83.5952] & [129.3098] & [334.8030] \\
\hline \multirow[t]{2}{*}{ Años 1992-1995 } & 1693.9423 & 2383.1818 & -689.2395 & 1113.5192 & 1618.5227 & -505.0035 \\
\hline & [80.8302] & [123.0965] & [267.9667] & [61.5097] & [92.3897] & [196.6566] \\
\hline \multirow[t]{5}{*}{ Diferencia } & 660.3654 & 817.9464 & -157.5810 & 447.6539 & 585.3491 & -137.6952 \\
\hline & [139.4708] & [348.3992] & [366.6935] & {$[90.4241]$} & [223.1823] & [235.3018] \\
\hline & \multicolumn{3}{|c|}{ Tasa Delitos Contra las Personas } & \multicolumn{3}{|c|}{ Tasa Homicidios Dolosos } \\
\hline & \multicolumn{3}{|c|}{ Exposición a la Reforma } & \multicolumn{3}{|c|}{ Exposición a la Reforma } \\
\hline & Alta & Baja & Diferencia & Alta & Baja & Diferencia \\
\hline \multirow[t]{2}{*}{ Años 1996-2000 } & 469.1154 & 588.9231 & -119.8077 & 9.9898 & 8.8756 & 1.1142 \\
\hline & [26.4127] & [36.7691] & [82.8970] & [0.2640] & [0.2861] & [2.6800] \\
\hline \multirow[t]{2}{*}{ Años 1992-1995 } & 365.9423 & 434.6591 & -68.7168 & 9.3088 & 9.3507 & -0.0418 \\
\hline & [16.2773] & [23.6583] & [64.1586] & [0.7825] & [0.8071] & [3.5264] \\
\hline \multirow[t]{2}{*}{ Diferencia } & 103.1731 & 154.2640 & -51.0909 & 0.6810 & {$[-0.4750$} & 1.1560 \\
\hline & [32.3345] & [67.8462] & [73.4689] & [1.3660] & [1.4112] & [1.9251] \\
\hline
\end{tabular}

Notas: Errores estándar entre corchetes, para las diferencias errores estándar agrupados a nivel provincial. Tasas de delitos cada 100 mil habitantes. Medida de exposición: Alta si la intensidad de adopción es Full desde el inicio; Baja si la implementación es Gradual o nunca la implementaron (Tabla A.2 del Apéndice A).

\section{Resultados}

Para obtener el efecto causal de la educación sobre los niveles de delitos se estima la ecuación (2) planteada en la Sección 4.5, considerando como variables de interés al total de hechos delictuosos, delitos contra la propiedad, contra las personas y homicidios dolosos (todos ellos expresados en tasas cada 100 mil habitantes). Se dispone de un panel de datos a nivel provincial que incluye características socioeconómicas de las provincias entre los años 1992 y 2008.

Para cada variable de interés se estima la ecuación mencionada, incorporando efectos fijos por provincia y año en todas las especificaciones. También se incorporan controles, y los que se consideran son: la tasa de pobreza de acuerdo a una línea de US\$2.5, el total de desocupación, el logaritmo de gasto provincial en educación básica (serie expresada a precios constantes del año 2001), la desigualdad de ingresos medida a través del coeficiente de Gini, la tasa de asistencia de educación de los niños entre 13 y 15 años y un índice de masculinidad entre los 15 y 24 años que mide la cantidad de hombres cada 100 mujeres.

Para darle mayor robustez a los resultados se emplean distintas medidas de la intensidad de la aplicación de la reforma. En el primer caso se considera una medida de "Grado de Implementación", que se obtiene al construirse una variable binaria que toma valor 1 en el caso que las provincias hayan adoptado la reforma intensa desde el principio (F) y 0 en otro caso (G o NI), Cuadro A.2 del Apéndice A. La segunda alternativa considerada fue construir una variable binaria 
indicativa de la adopción temprana o tardía de la LFE y se toma como referencia el año 1998 (dado que la implementación entre las provincias se dio entre 1996 y 2000). Aquellas que lo hicieron entre los años 1996 y 1997 se consideran adoptantes tempranas y el resto tardías (se construye una variable binaria que toma valor 1 en el primer caso y 0 en el segundo), como indicativo del grado de la exposición a la LFE (alta o baja). Finalmente la última variable considerada como alternativa a la intensidad de la reforma, considera el porcentaje de la implementación de la LFE en los establecimientos de cada provincia (Polimodal), el año que se toma como referencia es el 2000 ya que si bien muchas provincias implementaron la reforma previo a este año, el ciclo Polimodal no fue instrumentado rápidamente en todas ellas.

En el Cuadro 6 se reportan los resultados de las estimaciones de diferencias en diferencias para el total de hechos delictuosos. Se observa que la interacción entre una dummy que toma valor 1 para los años posteriores a la reforma y una medida de la intensidad de la aplicación de la misma que indica si la implementación fue generalizada desde el inicio (considerando las especificaciones con y sin controles socioeconómicos a nivel provincial que varían en el tiempo), el coeficiente de interés resulta negativo y estadísticamente significativo al 5 por ciento. Considerando adicionalmente otra medida de la intensidad de la reforma que indica una adopción temprana o tardía de acuerdo al año en el que cada provincia decidió acatarla (indicativo una alta o baja exposición a la ley), y tomando en cuenta especificaciones con y sin controles a nivel provincial (ambas con efectos fijos de año y provincia), el coeficiente estimado del término de la interacción resulta negativo y estadísticamente significativo al 5 y al 1 por ciento, respectivamente, dadas el resto de las variables incluidas en las estimaciones. Finalmente cuando se toma como medida de intensidad de la aplicación de la LFE al porcentaje de establecimientos Polimodal en el año 2000 en cada provincia, se observa que el coeficiente de la interacción es significativo al 5 por ciento en ambas especificaciones consideradas.

Estos resultados sugieren que la introducción de la reforma ocasionó una caída en el total de delitos para los años expuestos a la LFE. Los coeficientes estimados se encuentran en un rango de 953 y 1120 total de delitos menos (cada 100 mil habitantes) para el periodo afectado por la reforma educativa. Por ejemplo, en el caso que se considere el modelo que toma como medida de la intensidad de la reforma al grado de implementación, con controles, se observa que en todo el periodo post reforma y para las provincias que hayan realizado una implementación intensa desde el inicio, la caída estimada en el total de delitos es de 993.82 delitos cada 100 mil habitantes, dadas el resto de las variables incluidas en el modelo, con respecto a las provincias que implementaron la LFE de manera gradual o nunca lo hicieron. 
Cuadro 6

Diferencias en Diferencias para el Total de Hechos Delictuosos

\begin{tabular}{|c|c|c|c|c|c|c|}
\hline & \multicolumn{6}{|c|}{ Tasa Total Hechos Delictuosos } \\
\hline & \multicolumn{2}{|c|}{$\begin{array}{c}\text { Grado de } \\
\text { Implementación }\end{array}$} & \multicolumn{2}{|c|}{$\begin{array}{l}\text { Implementación } \\
\text { respecto a } 1998\end{array}$} & \multicolumn{2}{|c|}{$\begin{array}{l}\% \text { de Establecimientos } \\
\text { Polimodal en } 2000\end{array}$} \\
\hline & (1) & (2) & (1) & (2) & (1) & (2) \\
\hline \multirow[t]{2}{*}{ Interacción 1} & -1120.2528 & -993.8245 & & & & \\
\hline & {$[442.1042]^{\star *}$} & {$[356.8482]^{\star \star}$} & & & & \\
\hline \multirow[t]{2}{*}{ Interacción 2} & & & -985.1665 & -953.9622 & & \\
\hline & & & {$[395.9667]^{\star *}$} & {$[328.6682]^{\star * *}$} & & \\
\hline \multirow[t]{2}{*}{ Interacción 3} & & & & & -1015.2426 & -953.3107 \\
\hline & & & & & {$[380.4070]^{\star *}$} & {$[361.3904]^{* *}$} \\
\hline \multirow[t]{2}{*}{ Tasa de pobreza 2.5 US\$ } & & -4357.0794 & & -4124.4953 & & -2472.5756 \\
\hline & & {$[1,852.9230]^{\star *}$} & & {$[1,974.8924]^{\star \star}$} & & {$[1,398.0181]^{*}$} \\
\hline \multirow[t]{2}{*}{ Tasa de desocupación } & & -2561.0668 & & -3617.2862 & & -2275.4144 \\
\hline & & {$[2,650.5014]$} & & {$[2,891.3240]$} & & {$[3,197.5551]$} \\
\hline \multirow[t]{2}{*}{ Log. gasto educación básica } & & -991.8101 & & -707.7247 & & -430.1202 \\
\hline & & {$[452.4942]^{* *}$} & & [480.3425] & & [602.6036] \\
\hline \multirow[t]{2}{*}{ Desigualdad Gini } & & 837.5297 & & 737.7760 & & -946.1855 \\
\hline & & {$[2,798.5580]$} & & {$[2,817.3680]$} & & {$[2,296.2461]$} \\
\hline \multirow[t]{2}{*}{ Tasa de asistencia (13-15 años) } & & -2236.7190 & & -2813.9828 & & -2908.3984 \\
\hline & & {$[2,555.0341]$} & & {$[2,682.4706]$} & & {$[2,929.0807]$} \\
\hline \multirow[t]{2}{*}{ Índice de masculinidad (15-24 años) } & & -20.5569 & & -19.5102 & & -23.3357 \\
\hline & & [12.3424] & & [11.8132] & & {$[12.3587]^{*}$} \\
\hline \multirow[t]{2}{*}{ Constante } & 1527.8679 & 25858.2154 & 1525.4415 & 20613.7623 & 1526.9736 & 15929.3598 \\
\hline & {$[342.7403]^{\star \star *}$} & $10,053.6596]^{\star *}$ & {$[345.3522]^{* * \star}$} & ${ }^{*}[10,001.7251]^{*}$ & {$[340.4024]^{* * *}$} & * $[12,153.0978]$ \\
\hline Observaciones & 357 & 282 & 357 & 282 & 357 & 282 \\
\hline $\mathrm{R} 2$ & 0.86 & 0.91 & 0.86 & 0.91 & 0.86 & 0.91 \\
\hline
\end{tabular}

Fuente: Elaboración propia sobre la Base de Datos Socioeconómicos para América Latina y el CaribeSEDLAC CEDLAS y Banco Mundial (2012), DINIECE, MECON y SNIC. Notas: Errores estándar agrupados a nivel provincial entre corchetes. Tasas de delitos cada 100 mil habitantes. Todas las regresiones incluyen efectos fijos por año y provincia, y están ponderadas considerando el tamaño de población de cada una. Modelo (1) no incluye controles; Modelo (2) con controles. La variable Interacción 1 es la interacción entre la variable binaria que indica el periodo con tasas de delitos no afectadas por la reforma $1992-2000$ (igual a 0) y el que se considera con tasas de delitos afectadas por la misma (2003-2008), tomando valor 1 en este caso, con una variable binaria que es igual a 1 si la implementación fue full. En Interacción 2 se considera como medida de la intensidad a una variable binaria que vale 1 si la reforma se adoptó entre 1996 y 1997. Interacción 3 considera el porcentaje de establecimientos de educación media que adoptaron el Polimodal en 2000.

* significativo al 10\%; ** significativo al 5\%; *** significativo al $1 \%$.

En el Cuadro 7 se presentan los resultados de las estimaciones para los delitos contra la propiedad. En primer lugar se observa que la interacción entre la variable que indica el periodo afectado por la reforma y la intensidad medida a través de una dummy indicativa de una adopción generalizada desde el inicio, resulta negativa y estadísticamente significativa (al 5 por ciento en el modelo que no incluye controles socioeconómicos a nivel provincial y al 1 por ciento en el caso que los incorpora). Cuando la medida de intensidad empleada es una dummy que indica una adopción temprana versus una tardía (antes o después del año 1998), el coeficiente de la interacción estimado es negativo y significativo estadísticamente (al 5 por ciento en el modelo sin controles socioeconómicos provinciales y al 1 por ciento en el caso que los incorpora). Por último, cuando la intensidad de la reforma se mide a través del porcentaje de establecimientos Polimodal en el año 2000, el coeficiente 
estimado del término de la interacción resulta negativo y estadísticamente significativo al 1 por ciento, cuando la especificación incluye o no controles a nivel provincial.

En general, los resultados hallados para los delitos contra la propiedad indican una caída significativa de las tasas de este tipo de delitos ocasionada por la reforma. Las estimaciones indican una disminución en las tasas de delitos contra la propiedad cada 100 mil habitantes desde 738 a 866 , para el periodo expuesto a la reforma. Nuevamente, considerando el modelo que toma como medida de la intensidad de la reforma al grado de implementación, se observa que dadas el resto de las variables incluidas en la estimación, las tasas de delitos contra la propiedad disminuyen en 772.46 delitos contra la propiedad cada 100 mil habitantes en el periodo post reforma y para las provincias que la implementaron de manera intensa, con respecto a las provincias que lo hicieron de manera gradual o nunca la implementaron.

Cuadro 7

Diferencias en Diferencias para Delitos Contra la Propiedad

\begin{tabular}{|c|c|c|c|c|c|c|}
\hline & \multicolumn{6}{|c|}{ Tasa Delitos contra la Propiedad } \\
\hline & \multicolumn{2}{|c|}{$\begin{array}{c}\text { Grado de } \\
\text { Implementación }\end{array}$} & \multicolumn{2}{|c|}{$\begin{array}{l}\text { Implementación } \\
\text { respecto a } 1998\end{array}$} & \multicolumn{2}{|c|}{$\begin{array}{l}\% \text { de Establecimientos } \\
\text { Polimodal en } 2000\end{array}$} \\
\hline & $(1)$ & $(2)$ & $(1)$ & $(2)$ & $(1)$ & $(2)$ \\
\hline Interacción 1 & $\begin{array}{c}-866.6228 \\
{[327.6913]^{* *}}\end{array}$ & $\begin{array}{c}-772.4634 \\
{[272.1989]^{\star \star *}}\end{array}$ & & & & \\
\hline Interacción 2 & & & $\begin{array}{c}-738.0669 \\
{[300.8257]^{* *}}\end{array}$ & $\begin{array}{c}-722.7167 \\
{[251.5849]^{\star * *}}\end{array}$ & & \\
\hline Interacción 3 & & & & & $\begin{array}{c}-798.8465 \\
{[276.1560]^{\star \star \star}}\end{array}$ & $\begin{array}{c}-763.1754 \\
{[260.4800]^{* * *}}\end{array}$ \\
\hline Tasa de pobreza 2.5 US\$ & & $\begin{array}{c}-2942.9121 \\
{[1,684.6253]^{*}}\end{array}$ & & $\begin{array}{c}-2771.7633 \\
{[1,843.7310]}\end{array}$ & & $\begin{array}{c}-1428.2814 \\
{[1,424.0078]}\end{array}$ \\
\hline Tasa de desocupación & & $\begin{array}{c}-1511.6070 \\
{[1,870.5672]}\end{array}$ & & $\begin{array}{c}-2269.3909 \\
{[2,053.0913]}\end{array}$ & & $\begin{array}{l}-1333.1349 \\
{[2,308.8406]}\end{array}$ \\
\hline Log. gasto educación básica & & $\begin{array}{c}-848.2808 \\
{[354.5025]^{* *}}\end{array}$ & & $\begin{array}{l}-634.0983 \\
{[376.3606]}\end{array}$ & & $\begin{array}{c}-397.3880 \\
{[446.0868]}\end{array}$ \\
\hline Desigualdad Gini & & $\begin{array}{c}756.8141 \\
{[2,122.8393]}\end{array}$ & & $\begin{array}{c}641.1781 \\
{[2,104.4315]}\end{array}$ & & $\begin{array}{c}-623.7060 \\
{[1,743.4342]}\end{array}$ \\
\hline Tasa de asistencia (13-15 años) & & $\begin{array}{c}-1293.9092 \\
{[1,832.8368]}\end{array}$ & & $\begin{array}{c}-1748.4901 \\
{[1,958.8303]}\end{array}$ & & $\begin{array}{c}-1811.1998 \\
{[2,110.1207]}\end{array}$ \\
\hline Índice de masculinidad (15-24 años) & & $\begin{array}{l}-14.8583 \\
{[8.3628]^{\star}}\end{array}$ & & $\begin{array}{l}-14.0364 \\
{[8.0239]^{\star}}\end{array}$ & & $\begin{array}{l}-17.1170 \\
{[8.3172]^{\star}}\end{array}$ \\
\hline Constante & $\begin{array}{c}1004.3702 \\
{[252.5206]^{\star * *}}\end{array}$ & $\begin{array}{c}20804.7323 \\
{ }^{\star}[7,701.1640]^{\star \star}\end{array}$ & $\begin{array}{c}1002.5053 \\
{[255.8471]^{\star * \star}}\end{array}$ & $\begin{array}{c}16878.1377 \\
{[7,701.2765]^{\star \star}}\end{array}$ & $\begin{array}{c}1003.6919 \\
{[250.0969]^{\star \star \star}}\end{array}$ & $\begin{array}{l}12801.0121 \\
{[8,920.5041]}\end{array}$ \\
\hline Observaciones & 357 & 282 & 357 & 282 & 357 & 282 \\
\hline $\mathrm{R} 2$ & 0.87 & 0.91 & 0.86 & 0.91 & 0.86 & 0.91 \\
\hline
\end{tabular}

Elaboración propia sobre la Base de Datos Socioeconómicos para América Latina y el Caribe-SEDLAC, CEDLAS y Banco Mundial (2012), DINIECE, MECON y SNIC. Notas del Cuadro 6.

* significativo al $10 \%$; ** significativo al $5 \%$; *** significativo al $1 \%$.

En los Cuadros 8 y 9, se muestran los resultados de las estimaciones de diferencias en diferencias del impacto de la reforma educativa para las tasas de delitos contra las personas y los homicidios dolosos, respectivamente. En ambos casos puede observarse que para todas las opciones de intensidad de la aplicación de la reforma y para todas las especificaciones empleadas, no se encuentra evidencia que el coeficiente de interés, la interacción entre la variable Post y las tres variables alternativas de la intensidad de la aplicación, resulte estadísticamente significativo. 
Cuadro 8

Diferencias en Diferencias para Delitos Contra las Personas

\begin{tabular}{|c|c|c|c|c|c|c|}
\hline & \multicolumn{6}{|c|}{ Tasa Delitos contra las Personas } \\
\hline & \multicolumn{2}{|c|}{$\begin{array}{c}\text { Grado de } \\
\text { Implementación }\end{array}$} & \multicolumn{2}{|c|}{$\begin{array}{l}\text { Implementación } \\
\text { respecto a } 1998\end{array}$} & \multicolumn{2}{|c|}{$\begin{array}{c}\text { \% de Establecimientos } \\
\text { Polimodal en } 2000\end{array}$} \\
\hline & $(1)$ & $(2)$ & $(1)$ & $(2)$ & $(1)$ & $(2)$ \\
\hline Interacción 1 & $\begin{array}{l}-99.4347 \\
{[71.4129]}\end{array}$ & $\begin{array}{l}-73.1162 \\
{[58.2202]}\end{array}$ & & & & \\
\hline Interacción 2 & & & $\begin{array}{l}-75.3362 \\
{[64.0208]}\end{array}$ & $\begin{array}{l}-60.7011 \\
{[55.8062]}\end{array}$ & & \\
\hline Interacción 3 & & & & & $\begin{array}{l}-84.3079 \\
{[62.1257]}\end{array}$ & $\begin{array}{l}-77.6226 \\
{[63.4227]}\end{array}$ \\
\hline Tasa de pobreza 2.5 US\$ & & $\begin{array}{l}-747.8785 \\
{[483.9982]}\end{array}$ & & $\begin{array}{l}-735.6340 \\
{[481.0052]}\end{array}$ & & $\begin{array}{l}-592.4155 \\
{[363.7215]}\end{array}$ \\
\hline Tasa de desocupación & & $\begin{array}{l}-653.4176 \\
{[423.7727]}\end{array}$ & & $\begin{array}{l}-699.1965 \\
{[435.8697]}\end{array}$ & & $\begin{array}{l}-647.0897 \\
{[428.7102]}\end{array}$ \\
\hline Log. gasto educación básica & & $\begin{array}{c}-29.6917 \\
{[77.1691]}\end{array}$ & & $\begin{array}{c}-12.1403 \\
{[74.3804]}\end{array}$ & & $\begin{array}{c}16.4584 \\
{[86.7838]}\end{array}$ \\
\hline Desigualdad Gini & & $\begin{array}{c}93.4686 \\
{[416.1276]}\end{array}$ & & $\begin{array}{c}66.8746 \\
{[422.6899]}\end{array}$ & & $\begin{array}{c}-35.7718 \\
{[412.7390]}\end{array}$ \\
\hline Tasa de asistencia (13-15 años) & & $\begin{array}{l}-364.8048 \\
{[293.9936]}\end{array}$ & & $\begin{array}{l}-410.2535 \\
{[289.4077]}\end{array}$ & & $\begin{array}{l}-412.6083 \\
{[305.4098]}\end{array}$ \\
\hline Índice de masculinidad (15-24 años) & & $\begin{array}{l}-3.3238 \\
{[2.0903]}\end{array}$ & & $\begin{array}{l}-3.2426 \\
{[2.0102]}\end{array}$ & & $\begin{array}{l}-3.5616 \\
{[2.1151]}\end{array}$ \\
\hline Constante & $\begin{array}{c}279.7615 \\
{[43.0123]^{\star \star \star}}\end{array}$ & $\begin{array}{c}1655.9250 \\
{[1,528.6923]}\end{array}$ & $\begin{array}{c}279.5523 \\
{[43.9506]^{\star \star \star}}\end{array}$ & $\begin{array}{c}1345.7553 \\
{[1,436.5136]}\end{array}$ & $\begin{array}{c}279.6763 \\
{[43.2274]^{\star \star *}}\end{array}$ & $\begin{array}{c}828.8782 \\
{[1,747.4272]}\end{array}$ \\
\hline Observaciones & 357 & 282 & 357 & 282 & 357 & 282 \\
\hline $\mathrm{R} 2$ & 0.87 & 0.91 & 0.86 & 0.91 & 0.86 & 0.91 \\
\hline
\end{tabular}

Elaboración propia sobre la Base de Datos Socioeconómicos para América Latina y el Caribe-SEDLAC, CEDLAS y Banco Mundial (2012), DINIECE, MECON y SNIC. Notas del Cuadro 6.

* significativo al 10\%; ** significativo al 5\%; *** significativo al $1 \%$.

En general, los resultados encontrados en este trabajo coinciden con los efectos hallados por los trabajos previos, que analizan reformas educativas que alteran los años obligatorios de educación. En los mismos se encontraron reducciones significativas del total de delitos y los delitos contra la propiedad en particular, ya que la motivación subyacente de los mismos es de carácter económico y por ende susceptible de ser afectados por mayores logros educativos, si el retorno en el mercado laboral aumenta. Para los delitos contra las personas o los homicidios en particular, los resultados aquí obtenidos son los esperados en el modelo teórico del delito cuando se analizan estos tipos de crímenes, dado que la motivación subyacente de los mismos no tiene que ver principalmente con móviles económicos. 
Cuadro 9

Diferencias en Diferencias para Homicidios Dolosos

\begin{tabular}{|c|c|c|c|c|c|c|}
\hline & & & \multicolumn{2}{|c|}{ Tasa Homicidios Dolosos } & & \\
\hline & \multicolumn{2}{|c|}{$\begin{array}{c}\text { Grado de } \\
\text { Implementación }\end{array}$} & \multicolumn{2}{|c|}{$\begin{array}{l}\text { Implementación } \\
\text { respecto a } 1998\end{array}$} & \multicolumn{2}{|c|}{$\begin{array}{c}\% \text { de Establecimientos } \\
\text { Polimodal en } 2000\end{array}$} \\
\hline & $(1)$ & $(2)$ & $(1)$ & $(2)$ & $(1)$ & $(2)$ \\
\hline \multirow[t]{2}{*}{ Interacción 1} & -0.3295 & 0.1547 & & & & \\
\hline & {$[1.5425]$} & [1.4554] & & & & \\
\hline \multirow[t]{2}{*}{ Interacción 2} & & & 1.2305 & 1.1458 & & \\
\hline & & & [1.5868] & [1.4298] & & \\
\hline \multirow[t]{2}{*}{ Interacción 3} & & & & & -0.2755 & -0.7663 \\
\hline & & & & & [1.2569] & {$[0.9921]$} \\
\hline \multirow[t]{2}{*}{ Tasa de pobreza 2.5 US\$ } & & -6.7908 & & -7.2275 & & -5.1175 \\
\hline & & [8.0607] & & [7.6799] & & [6.2503] \\
\hline \multirow[t]{2}{*}{ Tasa de desocupación } & & 5.0265 & & 8.2753 & & 3.4043 \\
\hline & & [13.8973] & & [13.4339] & & [12.9893] \\
\hline \multirow[t]{2}{*}{ Log. gasto educación básica } & & 2.6674 & & 2.2372 & & 3.1964 \\
\hline & & [2.3463] & & [2.1106] & & [2.1379] \\
\hline \multirow[t]{2}{*}{ Desigualdad Gini } & & 13.9870 & & 11.7843 & & 14.6606 \\
\hline & & [12.2947] & & [11.8215] & & [13.8559] \\
\hline \multirow[t]{2}{*}{ Tasa de asistencia (13-15 años) } & & -0.4484 & & -0.6074 & & -0.1980 \\
\hline & & {$[5.5054]$} & & {$[6.2850]$} & & [5.4867] \\
\hline \multirow[t]{2}{*}{ Índice de masculinidad (15-24 años) } & & -0.0553 & & -0.0552 & & -0.0588 \\
\hline & & {$[0.0301]^{*}$} & & {$[0.0296]^{*}$} & & {$[0.0296]^{*}$} \\
\hline \multirow[t]{2}{*}{ Constante } & 9.0227 & -46.3693 & 9.0214 & -36.8551 & 9.0223 & -57.2093 \\
\hline & {$[0.8513]^{\star * *}$} & [46.5902] & {$[0.9012]^{\star * *}$} & [41.6964] & {$[0.8474]^{\star * *}$} & [43.1338] \\
\hline Observaciones & 356 & 281 & 356 & 281 & 356 & 281 \\
\hline $\mathrm{R} 2$ & 0.52 & 0.56 & 0.52 & 0.56 & 0.52 & 0.56 \\
\hline
\end{tabular}

Elaboración propia sobre la Base de Datos Socioeconómicos para América Latina y el Caribe-SEDLAC, CEDLAS y Banco Mundial (2012), DINIECE, MECON y SNIC. Notas del Cuadro 6.

* significativo al $10 \%$; ** significativo al $5 \%$; *** significativo al $1 \%$.

\section{Conclusiones}

En este trabajo se estima el efecto de la educación sobre los niveles de delitos para Argentina. Para evitar la endogeneidad existente entre las decisiones de involucrarse en actividades delictivas y las de educación, se utiliza una estrategia de identificación que emplea en la misma a la Ley Federal de Educación, reforma que aumentó los años de educación obligatorios en el país de manera exógena. La base de este trabajo se centra en el hecho que la reforma alteró efectivamente de manera exógena las decisiones de escolaridad a través de las distintas provincias. Dado que la implementación de la ley por parte de las distritos no se realizó de manera simultánea (llevándose a cabo entre los años 1996 y 2000) ni con la misma intensidad, se emplea este hecho para el diseño de la estrategia de identificación aquí empleada, utilizando una metodología quasi-experimental (diferencias en diferencias) para identificar los posibles cambios en las conductas criminales.

Los resultados obtenidos indican que para el total de los delitos denunciados y los delitos contra la propiedad en particular, la implementación de la LFE, ocasionó una caída significativa en los niveles de estos delitos para el periodo afectado por la reforma y las provincias que implementaron la reforma de manera intensa, temprana o con una mayor proporción de establecimientos Polimodal. No se encontró evidencia significativa en el caso de los delitos contra las personas y los homicidios, 
resultados consistentes con lo que se espera en el marco de la Teoría Económica del Delito, ya que en general se sostiene que estos delitos no son motivados por móviles económicos y por ende susceptibles de ser modificados por la educación de los individuos. Los resultados aquí encontrados son consistentes con los evidenciados por los trabajos previos que estudiaron la relación entre delitos y educación a través de la estimación del efecto causal.

Es importante destacar que en este trabajo solo se buscó captar el efecto neto de la educación sobre el delito, no intentando diferenciar cuales de los canales de la misma son los que realmente influyen en la disminución de las tasas registradas. Pero dados los periodos analizados y las características de la reforma se espera que sea el efecto capital humano el responsable de la disminución estimada en los niveles de delitos, ya que mayores años de educación generan mayores posibilidades de inserción en el mercado laboral y las oportunidades de involucrarse en actividades delictivas disminuyen, por aumentos en el costo de oportunidad. Pero no se descarta el hecho que el efecto incapacitación generado por la reforma también esté impactando sobre los resultados obtenidos, al obligar a más jóvenes a asistir a la escuela más años.

Este trabajo provee evidencia para un país en desarrollo como Argentina, del efecto de la educación sobre los delitos, coincidente con la ya encontrada para países desarrollados (Estados Unidos, Gran Bretaña y Suecia). Por lo que se puede afirmar que entre las políticas de seguridad tendientes a reducir los niveles de delitos, se debe considerar la importancia de la educación en el diseño de las mismas, ya que no solo genera retornos privados, si no también externalidades positivas tales como la disminución de los potenciales criminales en una sociedad. De modo que la inversión de los gobiernos en educación genera beneficios que no son tenidos en cuenta en su totalidad en el análisis costo-beneficio de tal inversión, y que resultan relevantes para toda la población, ya que además persisten en el tiempo. 


\section{Referencias}

Acevedo, C. (2008). "Los Costos Económicos de la Violencia en El Salvador". Universidad de Salamanca. América Latina Hoy, Vol. 50, pág. 71-88.

Alzúa, M. L. (2011). "Los Programas Sociales y el Delito contra la Propiedad: Evidencia del Programa Plan Jefes y Jefas de Hogar Desocupados en Argentina”. En: Perspectivas sobre el Desarrollo Seguridad Ciudadana y Bienestar vol. 9 No 1 . Corporación Andina de Fomento.

Angrist, J. y J. Pischcke (2009). "Mostly Harmless Econometrics: An Empiricist's Companion". Princeton University Press.

Alzúa, M., G. Bet, L. Gasparini y F. Haimovich (2010). “Assessing the Impact of Argentina's Ley Federal de Educación on Educational and Labor Outcomes". Poverty and Economic Policy (PEP) Research Report.

Berlinsky, S., S. Galiani y P. Gertler (2006). "The Effect of Pre-Primary Education on Primary School Performance”. William Davidson Institute Working Paper No 838.

Berthelon, M. y D. Kruger (2010). "Risky Behavior Among Youth: Incapacitation Effects of School on Adolescent Motherhood and Crime in Chile”. Journal of Public Economics 95, pág. 4153.

Bertrand, M., E. Duflo y S. Mullainathan (2004). "How Much Should We Trust Differences in differences Estimates?”. The Quarterly Journal of Economics, 119(1), pág. 249-275.

Black, S., P. Devereux y K. Salvanes (2008). "Staying in the Classroom and out of the Maternity Ward? The Effect of Compulsory Schooling Laws on Teenage Births". The Economic Journal 118, pág. 1025-1054.

Bourguignon, F. (1999). "Crime, Violence and Inequitable Development". Annual World Bank Conference on Development Economics, Washington, D.C., April 28-30.

Cerro, A.M. y O. Meloni (1999). "Desempleo, Distribución del Ingreso y Delincuencia en la Argentina”. Publicado en los Anales de la XXXIV Reunión de la Asociación Argentina de Economía Política.

Cerro, A. M. y A. Rodríguez Andrés (2011). "Typologies of Crime in the Argentine Provinces. A Panel Study 2000-2008". Publicado en los Anales de la XLVI Reunión Anual de la Asociación Argentina de Economía Política.

Chambouleyron, A., y M. Willington (1998). "Crimen y Castigo en la Argentina: un enfoque empírico”. Publicado en los Anales de la XXXII Reunión Anual de la Asociación Argentina de Economía Política.

Crosta, F. (2007). "Exploring the Effects of the School Levels Reform on Access and its Quality: The Education Federal Law of Argentina". Well-Being and Social Policy Magazine Vol. 3, № 1, pág. 97-122. Inter- American Conference on Social Security. 
Crosta, F. (2009). "Los Efectos de las Políticas Públicas sobre la Distribución del Ingreso. Evidencia para la Argentina”. Tesis de Doctorado. Universidad Nacional de La Plata.

Dirección Nacional de Información y Evaluación de la Calidad Educativa. Anuarios Estadísticos Educativos (1998-2006). Ministerio de Educación de la Nación Argentina.

Dirección Nacional de Política Criminal. Sistema Nacional de Información Criminal: Informes Total País y por Provincias (1992-2008). Ministerio de Justicia y Derechos Humanos de la Nación Argentina.

Dirección Nacional de Política Macroeconómica. Series de Gasto Público Consolidado (19932008). Ministerio de Economía y Finanzas Públicas de la Nación Argentina.

Di Tella, R. y E. Schargrodski (2004). "Do Police Reduce Crime? Estimates Using the Allocation of Police Forces after a Terrorist Attack". American Economic Review, Vol. 94 (1), pág. 115-133.

Di Tella, R., S. Edwards y E. Shargrodsky (2010). "The Economics of Crime Lessons For and From Latin America". Introducción. Eds. R. Di Tella, S. Edwards y E. Shargrodsky. NBER. The University of Chicago Press.

Fajnzylber, P., D. Lederman y N. Loayza (2002). "Inequality and Violent Crime”. Journal of Law and Economics, Vol. 45, $\mathrm{N}^{\mathrm{o}} 1$, Parte 1.

Freeman, R. (1999). "The Economics of Crime". Handbook of Labor Economics, Edition 1, Vol. 3, Capítulo 52, pág. 3529-3571. Elsevier.

Galiani, S. y E. Shargrodsky (2002). "Evaluating the Impact of School Decentralization on Educational Quality”. Journal of LACEA Economia, LACEA-Latin American and Caribbean Economic Association.

Grogger, J. (1998). "Market Wages and Youth Crime”. Journal of Labor Economics, Vol. 16, № 4, pág. 756-791.

Imbens, G. y J. Angrist (1994). "Identification and Estimation of Local Average Treatment Effects”. Econometrica 63(2), pág. 467-475.

Kelly, M. (2000). "Inequality and Crime". The Review of Economics and Statistics, Vol. 82, No 4, pág. 530-539.

Kessler, M, y A. Molinari (1997). "Una Aproximación Microeconómica al Crimen en la Argentina”. Publicado en los Anales de la XXXI Reunión Anual de la Asociación Argentina de Economía Política.

Laboratorio de Investigaciones sobre Crimen, Instituciones y Políticas-LICIP. Índice de Victimización Informe Mayo 2012.

Lochner, L. (2010). “Education Policy and Crime”. NBER. Working Paper 15894. 
Lochner, L. y Moretti, E. (2004). "The Effect of Education on Crime: Evidence from Prison Inmates, Arrests, and Self-reports". The American Economic Review 94, pág. 155-189.

Machin, S. y C. Meghir (2004). "Crime and Economic Incentives”. Journal of Human Resources 39 (4), pág.. 958-979.

Machin, S., O. Marie y S. Vujic (2010). "The Crime Reducing Effect of Education". IZA. Discussion Paper No. 5000.

Meghir, C., M. Palme y M. Schnabel (2012). "The Effect of Education Policy on Crime: An Intergenerational Perspective”. NBER Working Paper 18145.

Soares, R. (2010). "Welfare Costs of Crime and Common Violence: A Critical Review". Departamento de Economía. Pontificia Universidad de Rio. Trabajo para discusión $\mathrm{N}^{\mathrm{o}} 581$.

Soares, R. y J. Naritomi (2010). "Understanding High Crime Rates in Latin America: The Rol of Social and Policy Factors". Capítulo 1 en The Economics of Crime Lessons For and From Latin America. Eds. R. Di Tella, S. Edwards y E. Shargrodsky. NBER. The University of Chicago Press. 


\section{Apéndice A}

Cuadro A.1 Equivalencias de Niveles entre Sistemas Educativos

\begin{tabular}{|c|c|c|c|c|}
\hline \multicolumn{2}{|c|}{ Estructura Anterior } & \multirow{2}{*}{$\begin{array}{c}\text { Edades } \\
\text { aproximadas }\end{array}$} & \multicolumn{2}{|c|}{ Estructura LFE } \\
\hline Nivel & Años & & Años & Nivel \\
\hline \multirow{3}{*}{ Inicial } & $1^{\circ}$ & 3 & $1^{0}$ & \\
\hline & $2^{\circ}$ & 4 & $2^{\circ}$ & Inicial \\
\hline & $3^{\circ}$ & 5 & $3^{\circ}$ & \\
\hline \multirow{7}{*}{ Primario } & 10 & 6 & 10 & \multirow{3}{*}{ EGB1 } \\
\hline & $2^{\circ}$ & 7 & $2^{\circ}$ & \\
\hline & $3^{\circ}$ & 8 & $3^{\circ}$ & \\
\hline & $4^{\circ}$ & 9 & $4^{\circ}$ & \multirow{3}{*}{ EGB2 } \\
\hline & $5^{\circ}$ & 10 & $5^{\circ}$ & \\
\hline & $6^{\circ}$ & 11 & $6^{\circ}$ & \\
\hline & $7^{\circ}$ & 12 & $7^{\circ}$ & \multirow{3}{*}{ EGB3 } \\
\hline \multirow{5}{*}{ Secundario } & $11^{\circ}$ & 13 & $8^{\circ}$ & \\
\hline & $2^{0}$ & 14 & 9음 & \\
\hline & $3^{0}$ & 15 & $1^{0}$ & \multirow{3}{*}{ Polimoda } \\
\hline & $4^{\circ}$ & 16 & $2^{\circ}$ & \\
\hline & $5^{\circ}$ & 17 & $3^{\circ}$ & \\
\hline
\end{tabular}

Fuente: Ministerio de Educación de la Nación, DINIECE. Nota: los años obligatorios de cada sistema están sombreados. LFE: Ley Federal de Educación. EGB: Educación General Básica.

Cuadro A.2 Implementación de la Ley Federal de Educación en las Provincias Argentinas

\begin{tabular}{lcc}
\hline Provincia & $\begin{array}{c}\text { Año } \\
\text { implementación }\end{array}$ & $\begin{array}{c}\text { Modo de } \\
\text { Implementación }\end{array}$ \\
\hline Buenos Aires & 1996 & $\mathrm{~F}$ \\
Cdad. Autonoma de Bs. As. & & $\mathrm{NI}$ \\
Catamarca & 1999 & $\mathrm{G}$ \\
Chaco & 1997 & $\mathrm{G}$ \\
Chubut & 1999 & $\mathrm{G}$ \\
Córdoba & 1996 & $\mathrm{~F}$ \\
Corrientes & 1997 & $\mathrm{~F}$ \\
Entre Ríos & 1997 & $\mathrm{~F}$ \\
Formosa & 1998 & $\mathrm{~F}$ \\
Jujuy & 1998 & $\mathrm{G}$ \\
La Pampa & 1997 & $\mathrm{~F}$ \\
La Rioja & 1999 & $\mathrm{G}$ \\
Mendoza & 2000 & $\mathrm{G}$ \\
Misiones & 1998 & $\mathrm{~F}$ \\
Neuquén & 1998 & $\mathrm{G}$ \\
Río Negro & & $\mathrm{NI}$ \\
Salta & 1998 & $\mathrm{G}$ \\
San Juan & 1997 & $\mathrm{~F}$ \\
San Luis & 1998 & $\mathrm{~F}$ \\
Santa Cruz & 1998 & $\mathrm{~F}$ \\
Santa Fe & 1997 & $\mathrm{~F}$ \\
Santiago del Estero & 1998 & $\mathrm{~F}$ \\
Tucumán & 1998 & $\mathrm{~F}$ \\
Tierra del Fuego & 1998 & $\mathrm{G}$ \\
\hline & &
\end{tabular}

Fuente: Crosta (2009). Nota: Modo de Implementación F: Full desde el inicio; G: Gradual; NI: Nunca implementaron la reforma. 
Cuadro A.3 Hazard Model Implementación del 33 por ciento de Polimodal

\begin{tabular}{|c|c|c|c|c|c|c|c|c|}
\hline & \multicolumn{8}{|c|}{ Implementación de la Reforma 33\% de Polimodal } \\
\hline & $(1)$ & $(2)$ & (1) & $(2)$ & $(1)$ & $(2)$ & $(1)$ & $(2)$ \\
\hline Tasa hechos delictuosos & $\begin{array}{c}-0.0005 \\
{[0.0005]}\end{array}$ & $\begin{array}{c}-0.0006 \\
{[0.0003]^{*}}\end{array}$ & & & & & & \\
\hline Tasa contra las personas & & & $\begin{array}{c}-0.0035 \\
{[0.0042]}\end{array}$ & $\begin{array}{r}-0.0023 \\
{[0.0017]}\end{array}$ & & & & \\
\hline Tasa contra la propiedad & & & & & $\begin{array}{c}-0.0004 \\
{[0.0006]}\end{array}$ & $\begin{array}{c}-0.0006 \\
{[0.0004]}\end{array}$ & & \\
\hline Tasa homicidios dolosos & & & & & & & $\begin{array}{c}0.1207 \\
{[0.1228]}\end{array}$ & $\begin{array}{c}-0.0979 \\
{[0.2116]}\end{array}$ \\
\hline Tasa de pobreza 2.5 US\$ & $\begin{array}{c}8.5461 \\
{[6.0943]}\end{array}$ & $\begin{array}{c}13.4236 \\
{[6.5344]^{\star \star}}\end{array}$ & $\begin{array}{c}7.9251 \\
{[5.8851]}\end{array}$ & $\begin{array}{c}14.7026 \\
{[6.2375]^{\star \star}}\end{array}$ & $\begin{array}{c}9.8386 \\
{[6.1150]}\end{array}$ & $\begin{array}{c}15.0754 \\
{[6.3385]^{\star \star}}\end{array}$ & $\begin{array}{c}10.2417 \\
{[6.1083]^{\star}}\end{array}$ & $\begin{array}{c}19.2949 \\
{[6.2612]^{\star \star \star}}\end{array}$ \\
\hline Partido político & $\begin{array}{c}-0.3146 \\
{[0.6991]}\end{array}$ & $\begin{array}{c}-1.6604 \\
{[0.9144]^{\star}}\end{array}$ & $\begin{array}{c}0.2581 \\
{[0.7062]}\end{array}$ & $\begin{array}{c}-1.0204 \\
{[0.9744]}\end{array}$ & $\begin{array}{c}-0.2858 \\
{[0.6399]}\end{array}$ & $\begin{array}{c}-1.6846 \\
{[0.9680]^{*}}\end{array}$ & $\begin{array}{c}-0.0976 \\
{[0.6448]}\end{array}$ & $\begin{array}{c}-1.3103 \\
{[1.0612]}\end{array}$ \\
\hline Tasa de desocupación & $\begin{array}{c}3.2467 \\
{[9.0683]}\end{array}$ & $\begin{array}{l}-18.3260 \\
{[11.2437]}\end{array}$ & $\begin{array}{c}8.1428 \\
{[7.8119]}\end{array}$ & $\begin{array}{l}-11.7183 \\
{[9.9023]}\end{array}$ & $\begin{array}{c}3.3503 \\
{[10.0420]}\end{array}$ & $\begin{array}{l}-18.2475 \\
{[11.6688]}\end{array}$ & $\begin{array}{c}8.0666 \\
{[8.5857]}\end{array}$ & $\begin{array}{l}-11.1769 \\
{[11.9523]}\end{array}$ \\
\hline Log. gasto educación básica & $\begin{array}{c}0.5148 \\
{[0.3184]}\end{array}$ & $\begin{array}{c}1.2761 \\
{[0.5986]^{\star *}}\end{array}$ & $\begin{array}{c}0.2873 \\
{[0.2914]}\end{array}$ & $\begin{array}{c}1.0717 \\
{[0.5794]^{\star}}\end{array}$ & $\begin{array}{c}0.5690 \\
{[0.3082]^{\star}}\end{array}$ & $\begin{array}{c}1.3495 \\
{[0.6273]^{\star *}}\end{array}$ & $\begin{array}{c}0.4872 \\
{[0.2806]^{*}}\end{array}$ & $\begin{array}{c}1.4908 \\
{[0.9166]}\end{array}$ \\
\hline Desigualdad Gini & $\begin{array}{l}-12.3933 \\
{[21.6808]}\end{array}$ & $\begin{array}{c}-22.5146 \\
{[18.3419]}\end{array}$ & $\begin{array}{c}-20.8220 \\
{[21.9665]}\end{array}$ & $\begin{array}{l}-29.7118 \\
{[20.1609]}\end{array}$ & $\begin{array}{l}-13.8219 \\
{[22.4647]}\end{array}$ & $\begin{array}{c}-22.7991 \\
{[17.4525]}\end{array}$ & $\begin{array}{c}-17.0032 \\
{[21.9688]}\end{array}$ & $\begin{array}{c}-24.8776 \\
{[16.5580]}\end{array}$ \\
\hline Años prom. de educación hombres (15-24 años) & $\begin{array}{c}-0.0548 \\
{[1.1723]}\end{array}$ & $\begin{array}{c}-0.6479 \\
{[1.6911]}\end{array}$ & $\begin{array}{c}-0.0058 \\
{[1.1807]}\end{array}$ & $\begin{array}{c}-0.8384 \\
{[1.7281]}\end{array}$ & $\begin{array}{c}-0.2900 \\
{[1.1323]}\end{array}$ & $\begin{array}{c}-0.8413 \\
{[1.5664]}\end{array}$ & $\begin{array}{c}-0.3061 \\
{[0.9981]}\end{array}$ & $\begin{array}{c}-1.3772 \\
{[1.8498]}\end{array}$ \\
\hline Tasa de asistencia (13-15 años) & $\begin{array}{c}8.0576 \\
{[11.8718]}\end{array}$ & $\begin{array}{c}10.2389 \\
{[16.0816]}\end{array}$ & $\begin{array}{c}0.3794 \\
{[11.8514]}\end{array}$ & $\begin{array}{c}2.8505 \\
{[16.5631]}\end{array}$ & $\begin{array}{c}7.4060 \\
{[12.0958]}\end{array}$ & $\begin{array}{c}10.2750 \\
{[17.0545]}\end{array}$ & $\begin{array}{c}1.5679 \\
{[8.8902]}\end{array}$ & $\begin{array}{c}6.4670 \\
{[19.1019]}\end{array}$ \\
\hline Índice de masculinidad (15-24 años) & $\begin{array}{c}0.0271 \\
{[0.0412]}\end{array}$ & $\begin{array}{c}0.0630 \\
{[0.0483]}\end{array}$ & $\begin{array}{c}0.0236 \\
{[0.0424]}\end{array}$ & $\begin{array}{c}0.0628 \\
{[0.0496]}\end{array}$ & $\begin{array}{c}0.0300 \\
{[0.0423]}\end{array}$ & $\begin{array}{c}0.0607 \\
{[0.0458]}\end{array}$ & $\begin{array}{c}0.0345 \\
{[0.0440]}\end{array}$ & $\begin{array}{c}0.0526 \\
{[0.0418]}\end{array}$ \\
\hline Int & $\begin{array}{c}0.7121 \\
{[0.9742]}\end{array}$ & & $\begin{array}{c}0.9820 \\
{[1.1897]}\end{array}$ & & $\begin{array}{c}0.5273 \\
{[0.9445]}\end{array}$ & & $\begin{array}{c}0.3968 \\
{[0.8972]}\end{array}$ & \\
\hline d2 & & $\begin{array}{c}2.9887 \\
{[2.2185]}\end{array}$ & & $\begin{array}{c}2.8062 \\
{[2.1320]}\end{array}$ & & $\begin{array}{c}2.9636 \\
{[2.2051]}\end{array}$ & & $\begin{array}{c}2.8323 \\
{[2.2890]}\end{array}$ \\
\hline d3 & & $\begin{array}{c}2.9209 \\
{[2.5109]}\end{array}$ & & $\begin{array}{c}2.5500 \\
{[2.3252]}\end{array}$ & & $\begin{array}{c}2.7703 \\
{[2.5602]}\end{array}$ & & $\begin{array}{c}2.2101 \\
{[2.5996]}\end{array}$ \\
\hline d4 & & $\begin{array}{c}4.9428 \\
{[2.5526]^{*}}\end{array}$ & & $\begin{array}{c}4.5110 \\
{[2.3438]^{\star}}\end{array}$ & & $\begin{array}{c}4.6238 \\
{[2.6188]^{\star}}\end{array}$ & & $\begin{array}{c}3.6778 \\
{[2.4297]}\end{array}$ \\
\hline d5 & & $\begin{array}{c}2.6396 \\
{[2.2574]}\end{array}$ & & $\begin{array}{c}2.0715 \\
{[2.0277]}\end{array}$ & & $\begin{array}{c}2.1533 \\
{[2.3994]}\end{array}$ & & $\begin{array}{c}0.7211 \\
{[2.1176]}\end{array}$ \\
\hline d6 & & $\begin{array}{c}5.4729 \\
{[3.2737]^{*}}\end{array}$ & & $\begin{array}{c}5.1628 \\
{[3.1191]^{\star}}\end{array}$ & & $\begin{array}{c}5.3316 \\
{[3.3188]}\end{array}$ & & $\begin{array}{c}5.0010 \\
{[3.7629]}\end{array}$ \\
\hline Constante & $\begin{array}{c}-16.1037 \\
{[18.1116]}\end{array}$ & $\begin{array}{l}-25.0045 \\
{[20.2328]}\end{array}$ & $\begin{array}{c}-1.3156 \\
{[15.9148]}\end{array}$ & $\begin{array}{l}-10.5880 \\
{[18.6978]}\end{array}$ & $\begin{array}{c}-14.7024 \\
{[18.5915]}\end{array}$ & $\begin{array}{c}-25.0141 \\
{[21.7479]}\end{array}$ & $\begin{array}{c}-8.8349 \\
{[14.9645]}\end{array}$ & $\begin{array}{l}-19.5411 \\
{[24.8636]}\end{array}$ \\
\hline Observaciones & 102 & 102 & 102 & 102 & 102 & 102 & 101 & 101 \\
\hline
\end{tabular}

Notas: Tasas de delitos cada 100 mil habitantes. Errores estándar agrupados a nivel provincial en corchetes. (1) Modelo con tendencia temporal; (2) Modelo con dummies de tiempo. Ponderación por población provincial.

* significativo al 10\%; ** significativo al 5\%; *** significativo al $1 \%$. 
Cuadro A. 4 Hazard Model Implementación del 66 por ciento de Polimodal

\begin{tabular}{|c|c|c|c|c|c|c|c|c|}
\hline & \multicolumn{8}{|c|}{ Implementación de la Reforma $66 \%$ de Polimodal } \\
\hline & (1) & (2) & (1) & (2) & (1) & (2) & (1) & (2) \\
\hline Tasa hechos delictuosos & $\begin{array}{l}-0.0003 \\
{[0.0003]}\end{array}$ & $\begin{array}{c}-0.0007 \\
{[0.0003]^{\star *}}\end{array}$ & & & & & & \\
\hline Tasa contra las personas & & & $\begin{array}{l}-0.0017 \\
{[0.0026]}\end{array}$ & $\begin{array}{l}-0.0028 \\
{[0.0017]}\end{array}$ & & & & \\
\hline Tasa contra la propiedad & & & & & $\begin{array}{l}-0.0002 \\
{[0.0004]}\end{array}$ & $\begin{array}{c}-0.0007 \\
{[0.0004]^{*}}\end{array}$ & & \\
\hline Tasa homicidios dolosos & & & & & & & $\begin{array}{c}0.1737 \\
{[0.1564]}\end{array}$ & $\begin{array}{c}0.1393 \\
{[0.1491]}\end{array}$ \\
\hline Tasa de pobreza 2.5 US $\$$ & $\begin{array}{c}3.2595 \\
{[3.4862]}\end{array}$ & $\begin{array}{c}6.1061 \\
{[4.2047]}\end{array}$ & $\begin{array}{c}2.6484 \\
{[4.0668]}\end{array}$ & $\begin{array}{c}7.4308 \\
{[4.1932]^{*}}\end{array}$ & $\begin{array}{c}4.0064 \\
{[3.5417]}\end{array}$ & $\begin{array}{c}7.5738 \\
{[4.3670]^{*}}\end{array}$ & $\begin{array}{c}3.3718 \\
{[4.2006]}\end{array}$ & $\begin{array}{c}9.0584 \\
{[4.0216]^{* *}}\end{array}$ \\
\hline Partido político & $\begin{array}{c}0.3003 \\
{[0.7189]}\end{array}$ & $\begin{array}{c}-0.1646 \\
{[0.9865]}\end{array}$ & $\begin{array}{c}0.6740 \\
{[0.8478]}\end{array}$ & $\begin{array}{c}0.6506 \\
{[1.3155]}\end{array}$ & $\begin{array}{c}0.3282 \\
{[0.6673]}\end{array}$ & $\begin{array}{c}-0.2626 \\
{[0.9105]}\end{array}$ & $\begin{array}{c}0.5176 \\
{[0.7327]}\end{array}$ & $\begin{array}{c}0.0867 \\
{[1.0564]}\end{array}$ \\
\hline Tasa de desocupación & $\begin{array}{c}7.3199 \\
{[10.8187]}\end{array}$ & $\begin{array}{c}-28.3172 \\
{[12.8811]^{\star *}}\end{array}$ & $\begin{array}{c}9.7006 \\
{[10.4640]}\end{array}$ & $\begin{array}{c}-21.4549 \\
{[10.6929]^{\star \star}}\end{array}$ & $\begin{array}{c}7.2806 \\
{[11.4205]}\end{array}$ & $\begin{array}{c}-26.9557 \\
{[15.0044]^{*}}\end{array}$ & $\begin{array}{c}9.2143 \\
{[10.6680]}\end{array}$ & $\begin{array}{c}-16.0029 \\
{[11.5605]}\end{array}$ \\
\hline Log. gasto educación básica & $\begin{array}{c}0.5462 \\
{[0.2999]^{\star}}\end{array}$ & $\begin{array}{c}1.7870 \\
{[0.6651]^{\star \star \star}}\end{array}$ & $\begin{array}{c}0.4206 \\
{[0.3239]}\end{array}$ & $\begin{array}{c}1.5483 \\
{[0.6212]^{\star \star}}\end{array}$ & $\begin{array}{c}0.5853 \\
{[0.3015]^{\star}}\end{array}$ & $\begin{array}{c}1.8240 \\
{[0.7022]^{\star \star \star}}\end{array}$ & $\begin{array}{c}0.4423 \\
{[0.2684]^{\star}}\end{array}$ & $\begin{array}{c}1.5333 \\
{[0.5904]^{\star \star \star}}\end{array}$ \\
\hline Desigualdad Gini & $\begin{array}{c}-8.2270 \\
{[20.3221]}\end{array}$ & $\begin{array}{c}-20.3489 \\
{[16.3344]}\end{array}$ & $\begin{array}{l}-12.1503 \\
{[19.4149]}\end{array}$ & $\begin{array}{c}-31.0812 \\
{[18.3108]^{*}}\end{array}$ & $\begin{array}{c}-9.3076 \\
{[21.0632]}\end{array}$ & $\begin{array}{c}-20.1859 \\
{[17.0126]}\end{array}$ & $\begin{array}{l}-13.8679 \\
{[21.5502]}\end{array}$ & $\begin{array}{c}-28.2382 \\
{[20.4053]}\end{array}$ \\
\hline Años prom. de educación hombres (15-24 años) & $\begin{array}{c}0.1325 \\
{[1.0422]}\end{array}$ & $\begin{array}{c}0.1866 \\
{[1.9487]}\end{array}$ & $\begin{array}{c}0.1122 \\
{[1.0084]}\end{array}$ & $\begin{array}{c}-0.0371 \\
{[2.0814]}\end{array}$ & $\begin{array}{l}-0.0059 \\
{[1.0195]}\end{array}$ & $\begin{array}{l}-0.1086 \\
{[1.7537]}\end{array}$ & $\begin{array}{c}0.0706 \\
{[0.8603]}\end{array}$ & $\begin{array}{c}-0.4135 \\
{[1.9743]}\end{array}$ \\
\hline Tasa de asistencia (13-15 años) & $\begin{array}{l}-2.2878 \\
{[9.6823]}\end{array}$ & $\begin{array}{c}-7.3540 \\
{[18.5945]}\end{array}$ & $\begin{array}{c}-6.8500 \\
{[11.4029]}\end{array}$ & $\begin{array}{l}-16.9405 \\
{[20.0105]}\end{array}$ & $\begin{array}{c}-2.7326 \\
{[9.4772]}\end{array}$ & $\begin{array}{c}-6.1695 \\
{[19.3131]}\end{array}$ & $\begin{array}{l}-8.2455 \\
{[9.1498]}\end{array}$ & $\begin{array}{c}-14.7146 \\
{[19.6753]}\end{array}$ \\
\hline Índice de masculinidad (15-24 años) & $\begin{array}{c}0.0538 \\
{[0.0485]}\end{array}$ & $\begin{array}{c}0.1282 \\
{[0.0553]^{\star *}}\end{array}$ & $\begin{array}{c}0.0525 \\
{[0.0481]}\end{array}$ & $\begin{array}{c}0.1306 \\
{[0.0654]^{\star *}}\end{array}$ & $\begin{array}{c}0.0562 \\
{[0.0500]}\end{array}$ & $\begin{array}{c}0.1211 \\
{[0.0521]^{\star \star}}\end{array}$ & $\begin{array}{c}0.0587 \\
{[0.0481]}\end{array}$ & $\begin{array}{c}0.0996 \\
{[0.0579]^{*}}\end{array}$ \\
\hline Int & $\begin{array}{c}0.4029 \\
{[0.7632]}\end{array}$ & & $\begin{array}{c}0.5216 \\
{[0.8970]}\end{array}$ & & $\begin{array}{c}0.3362 \\
{[0.7484]}\end{array}$ & & $\begin{array}{c}0.4604 \\
{[0.8797]}\end{array}$ & \\
\hline d2 & & $\begin{array}{c}4.0744 \\
{[2.3197]^{*}}\end{array}$ & & $\begin{array}{c}3.8316 \\
{[2.1064]^{*}}\end{array}$ & & $\begin{array}{c}4.0069 \\
{[2.3975]^{\star}}\end{array}$ & & $\begin{array}{c}3.3417 \\
{[1.9868]^{*}}\end{array}$ \\
\hline d3 & & $\begin{array}{c}4.6028 \\
{[2.7588]^{\star}}\end{array}$ & & $\begin{array}{c}4.2812 \\
{[2.4079]^{\star}}\end{array}$ & & $\begin{array}{c}4.3329 \\
{[2.8749]}\end{array}$ & & $\begin{array}{c}3.2857 \\
{[2.1138]}\end{array}$ \\
\hline d4 & & $\begin{array}{c}7.7220 \\
{[3.0867]^{\star \star}}\end{array}$ & & $\begin{array}{c}7.3717 \\
{[2.6841]^{\star \star \star}}\end{array}$ & & $\begin{array}{c}7.2804 \\
{[3.2238]^{\star \star}}\end{array}$ & & $\begin{array}{c}5.9645 \\
{[2.1372]^{\star \star \star}}\end{array}$ \\
\hline d6 & & $\begin{array}{c}5.6909 \\
{[4.1479]}\end{array}$ & & $\begin{array}{c}5.5198 \\
{[3.7505]}\end{array}$ & & $\begin{array}{c}5.4933 \\
{[4.1486]}\end{array}$ & & $\begin{array}{c}4.6966 \\
{[3.5251]}\end{array}$ \\
\hline d7 & & $\begin{array}{c}2.7810 \\
{[2.5188]}\end{array}$ & & $\begin{array}{c}2.6849 \\
{[2.4305]}\end{array}$ & & $\begin{array}{c}2.6774 \\
{[2.4811]}\end{array}$ & & $\begin{array}{c}2.7235 \\
{[2.1165]}\end{array}$ \\
\hline d8 & & $\begin{array}{c}2.3161 \\
{[2.4287]}\end{array}$ & & $\begin{array}{c}2.6653 \\
{[2.2976]}\end{array}$ & & $\begin{array}{c}2.2328 \\
{[2.3109]}\end{array}$ & & $\begin{array}{c}2.5465 \\
{[2.0723]}\end{array}$ \\
\hline Constant & $\begin{array}{l}-14.3092 \\
{[16.6194]}\end{array}$ & $\begin{array}{c}-34.2674 \\
{[19.4919]^{\star}}\end{array}$ & $\begin{array}{c}-5.8624 \\
{[15.7704]}\end{array}$ & $\begin{array}{c}-15.6031 \\
{[15.6533]}\end{array}$ & $\begin{array}{c}-13.5467 \\
{[17.3785]}\end{array}$ & $\begin{array}{c}-33.5003 \\
{[22.4986]}\end{array}$ & $\begin{array}{c}-6.4786 \\
{[14.6813]}\end{array}$ & $\begin{array}{c}-14.5243 \\
{[16.7111]}\end{array}$ \\
\hline Observations & 127 & 127 & 127 & 127 & 127 & 127 & 126 & 126 \\
\hline
\end{tabular}

Notas: Tasas de delitos cada 100 mil habitantes. Errores estándar agrupados a nivel provincial en corchetes. (1) Modelo con tendencia temporal; (2) Modelo con dummies de tiempo. Ponderación por población provincial.

* significativo al 10\%; ** significativo al 5\%; *** significativo al $1 \%$. 